MATHEMATICS OF COMPUTATION

Volume 73 , Number 245 , Pages $333-357$

S 0025-5718(03)01567-9

Article electronically published on July 7, 2003

\title{
LINEAR ALGEBRA ALGORITHMS FOR DIVISORS ON AN ALGEBRAIC CURVE
}

\author{
KAMAL KHURI-MAKDISI
}

\begin{abstract}
We use an embedding of the symmetric $d$ th power of any algebraic curve $C$ of genus $g$ into a Grassmannian space to give algorithms for working with divisors on $C$, using only linear algebra in vector spaces of dimension $O(g)$, and matrices of size $O\left(g^{2}\right) \times O(g)$. When the base field $k$ is finite, or if $C$ has a rational point over $k$, these give algorithms for working on the Jacobian of $C$ that require $O\left(g^{4}\right)$ field operations, arising from the Gaussian elimination. Our point of view is strongly geometric, and our representation of points on the Jacobian is fairly simple to deal with; in particular, none of our algorithms involves arithmetic with polynomials. We note that our algorithms have the same asymptotic complexity for general curves as the more algebraic algorithms in Florian Hess' 1999 Ph.D. thesis, which works with function fields as extensions of $k[x]$. However, for special classes of curves, Hess' algorithms are asymptotically more efficient than ours, generalizing other known efficient algorithms for special classes of curves, such as hyperelliptic curves (Cantor 1987), superelliptic curves (Galbraith, Paulus, and Smart 2002), and $C_{a b}$ curves (Harasawa and Suzuki 2000); in all those cases, one can attain a complexity of $O\left(g^{2}\right)$.
\end{abstract}

\section{INTRODUCTION}

Let $C$ be a smooth algebraic curve of genus $g$ over a field $k$, and assume for simplicity in this introduction that $C$ has a rational point over $k$. When $g=1, C$ is an elliptic curve, and can be represented as a plane cubic; this is the Weierstrass model. In that case, the group law on $C$ is easy to describe and to implement, and has led to many effective algorithms in cryptography that make use of the difficulty of solving the discrete logarithm problem in the group of $k$-rational points of $C$ when $k$ is a finite field. If the genus of $C$ is larger, then one can try to work with the group law on the Jacobian of $C$. If $C$ admits a morphism of small degree to $\mathbf{P}^{1}$ (for instance, if $C$ is hyperelliptic), and if the genus $g$ is large compared to the order of $k$, then subexponential algorithms are known for the discrete logarithm on the Jacobian; see ADH94] for hyperelliptic curves, GPS02 for superelliptic curves, and Hes99] for general curves. Computing in the Jacobian of a large genus curve is nonetheless of intrinsic interest.

One problem is that for large $g$, the Jacobian is somewhat difficult to describe directly as an algebraic variety. For instance, if one wishes to embed the Jacobian into projective space, the standard way is to take a very ample line bundle (say, 3 or 4 times the theta-divisor), which leads to an embedding of the Jacobian into

Received by the editor November 7, 2001 and, in revised form, March 29, 2002.

2000 Mathematics Subject Classification. Primary 11Y16, 14Q05, 14H40, 11 G20. 
a projective space of exponentially large dimension (e.g., $3^{g}-1$ or $4^{g}-1$ ). This is not practical for explicit computations, especially as the equations defining the group law on the Jacobian will probably be similarly intractable. The other way to deal with the Jacobian is to use the fact that it is essentially an ideal class group attached to the function field of $C$; a divisor is a certain kind of ideal, and linear equivalence between divisors is equivalence in the ideal class group. Thus one works directly with divisors (or ideals) on $C$, and spends time mostly on finding appropriate elements of the function field in order to reduce divisors to a canonical form modulo linear equivalence. If the curve $C$ is hyperelliptic, this is relatively easy, since the Jacobian is analogous to the ideal class group of a quadratic field; questions about the Jacobian can be translated into questions about binary quadratic forms over the polynomial ring $k[x]$. This allows one to implement the group operations on the Jacobian of a hyperelliptic curve in "time" proportional to $g^{2}$, as in Can87. By this we mean that the algorithms require $O\left(g^{2}\right)$ operations in the field $k$. The articles [GPS02] and [HSO0] give $O\left(g^{2}\right)$ algorithms for a wider class of curves, the superelliptic and $C_{a b}$ curves, using more sophisticated techniques to work with the ideal classes; the implied constants are however quite large, and there is some possibility that they could grow with the genus $g$. As for a general curve of genus $g$, there are algorithms by F. Hess (see [Hes99] and [Hes02]) which both implement the group operations in the Jacobian, and determine its group structure over a finite field. Hess' algorithms begin with a map of degree $n$ from the curve $C$ to $\mathbf{P}^{1}$, and work with ideals in the function field of $C$, viewed as a degree $n$ extension of $k(x)$. If $n$ is fixed or bounded, then Hess' algorithms require $O\left(g^{2}\right)$ field operations to implement the group law on the Jacobian. This generalizes the previously mentioned results on hyperelliptic, superelliptic, and $C_{a b}$ curves. However, the minimum possible $n$ for a general curve of genus $g$ is approximately $g / 2$ (see [GH94], p. 261). In this case (and, more generally, for $n=O(g)$ ), the complexity of Hess' algorithms rises to $O\left(g^{4}\right)$ field operations per group operation in the Jacobian. Besides Hess' algorithms for general curves, we note two earlier geometric algorithms that are due to Huang and Ierardi [HI94] and Volcheck Vol94. Both algorithms rely on a description of $C$ as a plane curve with singularities, and the steps can become fairly involved (approximately $O\left(g^{7}\right)$ field operations are needed per group operation in the Jacobian).

In this paper, we give a set of straightforward geometric algorithms for the Jacobian of $C$ that involve only $O\left(g^{4}\right)$ field operations. Moreover, our algorithms are quite simple to implement, as they only involve linear algebra (row reduction) on matrices of size $O\left(g^{2}\right) \times O(g)$, and in vector spaces of dimension $O(g)$. In particular, no polynomial algebra is ever used explicitly ( a fortiori, no Gröbner bases or field extensions either). One can however argue that the representation of $C$ that we use implicitly works with quadratic polynomials in $O(g)$ variables. Specifically, we take a line bundle $\mathcal{L}$ on $C$, whose degree $N$ is approximately $6 g$, and work with the projective representation of $C$ arising from $H^{0}(C, \mathcal{L})$. In our setting, the homogeneous ideal defining $C$ is generated by quadratic polynomials (elements of $\left.\operatorname{Sym}^{2} H^{0}(C, \mathcal{L})\right)$, which can be thought of as the kernel of the multiplication map

$$
\mu: H^{0}(C, \mathcal{L}) \otimes H^{0}(C, \mathcal{L}) \rightarrow \operatorname{Sym}^{2} H^{0}(C, \mathcal{L}) \rightarrow H^{0}\left(C, \mathcal{L}^{\otimes 2}\right) .
$$

We note that the dimensions of the vector spaces $H^{0}(C, \mathcal{L})$ and $H^{0}\left(C, \mathcal{L}^{\otimes 2}\right)$ are both $O(g)$, being approximately $5 g$ and $11 g$, respectively. Then $\mu$ can be described in terms of any suitable bases for these vector spaces. This article does not present 
any new ideas on how to efficiently construct curves $C$ represented in the above form, and, what is more, on how to construct curves along with some nontrivial elements of their Jacobians. Nonetheless, the first question is easy to answer in principle: for example, choose a polynomial $f(x, y)$ which describes a plane curve with singularities that is a projection of $C$, and which passes through a given nonsingular point $P_{0}$ such as $(0,0)$; then we can let $\mathcal{L}=\mathcal{O}_{C}\left(N P_{0}\right)$, and use the algorithms of Hes99] and of Hes02] (or perhaps those of [HI94 or of [Vol94]) to compute the global sections of $\mathcal{L}$ and of $\mathcal{L}^{\otimes 2}$, and hence the map $\mu$. This only needs to be done once, in obtaining the initial description of $C$ in the form suitable for our algorithms. As for the second question above, we have not come up with any interesting methods to produce divisors on our curve $C$ corresponding to nontrivial points on the Jacobian. The best that we can do is to suggest choosing the plane curve to pass through a small number of given points $P_{1}, \ldots, P_{n}$, with $n$ small compared to $g$. Then we can obtain divisors generated by $P_{1}, \ldots, P_{n}$, but these divisors may not be "typical," for all we know. Another topic that we have not addressed (but which is covered in [Hes99]) is the question of efficiently computing the order, or, even better, the group structure, of the Jacobian over a finite field.

The basic forms of our algorithms, given in Section 4, are fairly easy to describe and to understand. The algorithms involve only subspaces of the two vector spaces $H^{0}(C, \mathcal{L})$ and $H^{0}\left(C, \mathcal{L}^{\otimes 2}\right)$ and the map $\mu$, and require one to solve various systems of linear equations in this setting. In Section 5, and to a lesser extent in Section 4 we give some faster, but slightly more complicated, algorithms for working with divisors on $C$ and with the Jacobian, including an algorithm that essentially solves the general Riemann-Roch problem for a divisor (written as a difference of effective divisors) $D_{1}-D_{2}$ in time $O\left(\max \left(g, \operatorname{deg} D_{1}, \operatorname{deg} D_{2}\right)^{4}\right)$. These faster algorithms still require time $O\left(g^{4}\right)$, but work with vector spaces of smaller dimension; for example, we can get away with taking the line bundle $\mathcal{L}$ to have degree approximately $3 g$, but then need to work (implicitly) with polynomials of higher degree in the projective embedding of $C$. Nonetheless, the structure of our faster algorithms is virtually identical to that of the basic forms of our algorithms. Our results follow from three main insights:

(1) We use a simple representation for effective divisors $D$ on $C$, in terms of the projective embedding given by the line bundle $\mathcal{L}$ above. Namely, so long as the degree $d$ of $D$ is small compared to that of $\mathcal{L}$, we can identify $D$ uniquely by the linear subspace $W_{D}^{*}$ of projective space that is spanned by $D$ (in a way that makes sense even if $D$ has points of multiplicity greater than 1). Dually, we represent $D$ by the space $W_{D} \subset H^{0}(C, \mathcal{L})$ consisting of global sections $s \in H^{0}(C, \mathcal{L})$ that vanish at $D$. Since the effective divisor $D$ corresponds to a point on the symmetric power $\mathrm{Sym}^{d} C$, we are essentially working with an embedding of $\operatorname{Sym}^{d} C$ into the Grassmannian parametrizing codimension $d$ subspaces of $H^{0}(C, \mathcal{L})$. As discussed in Section 2 below, this is much better than passing to a projective model of $\mathrm{Sym}^{d} C$ using the Plücker embedding. Perhaps other computational issues in algebraic geometry can become simpler if one is willing to work more extensively with Grassmannians and linear algebra, instead of always sticking to projective space and polynomials. The author hopes that this article will promote further investigation into the topic. 
(2) We no longer insist on reducing divisors to a canonical form, but instead represent an element of the Jacobian as an effective divisor of a certain degree $d \geq g+1$. The basic form of our algorithms in Section 4 can use any $d \geq 2 g+1$, and an improvement in Section 5 uses $d \geq g+1$. It is quite doable to bring this down to $d=g$, but at the expense of some complications in the algorithms. As a result, a given element of the Jacobian can be represented by many different subspaces $W_{D}$, where only the linear equivalence class of $D$ is well-defined. We of course give an algorithm to determine when two divisors represent the same point in the Jacobian; it also takes time $O\left(g^{4}\right)$. (More precisely, our algorithms take time $O\left(\max (d, g)^{4}\right)$, in case someone should wish to work with divisors of extremely large degree.)

(3) We work in terms of multiplication of sections of line bundles, as well as a form of division (Lemma 2.2 and Lemma/Algorithm 2.3). This amounts to working directly in the projective coordinate ring of $C$, instead of the full polynomial algebra of the projective space in which $C$ lies. This produces a substantial time savings, since for fixed $\ell$ and large $g$, the space of degree $\ell$ polynomials has dimension $O\left(\operatorname{dim} V^{\ell}\right)=O\left(g^{\ell}\right)$, while the restriction of such polynomials to $C$ is the space $H^{0}\left(C, \mathcal{L}^{\otimes \ell}\right)$, whose dimension is $O(\ell g)$. Moreover, the multiplication and division operations are the basis of our addition and flipping algorithms (Theorems/Algorithms 3.6 and 3.10), which are fundamental to our work. The flipping algorithm is especially important: given a divisor $D$ on $C$, and a hyperplane containing $D$, it allows us to find the "complementary" divisor $D^{\prime}$ such that $D+D^{\prime}$ is the intersection of $C$ with the hyperplane in question. The algorithm is inspired by geometric considerations, as explained in the discussion preceding Theorem/Algorithm 3.10 The flipping algorithm is what allows us to pass to divisor classes; for example, flipping twice with respect to different hyperplanes replaces $D$ with another divisor linearly equivalent to it.

We conclude this introduction by pointing out the connection between our way of representing divisors, and the way in Cantor's algorithm for hyperelliptic curves; it should be possible to say something similar for superelliptic and $C_{a b}$ curves. For simplicity, we restrict to the case where $k$ does not have characteristic 2 , and so consider a curve $C: y^{2}=f(x)$, where $f(x) \in k[x]$ has degree $2 g+1$. Then a reduced (effective) divisor $D$ of degree $d \leq g$ is represented in Cantor's algorithm by the pair of polynomials $(a(x), b(x))$, with $a(x)$ monic of degree $d, b(x)$ of degree at most $d-1$, and $b(x)^{2} \equiv f(x)(\bmod a(x))$. If we factor $a(x)=\prod_{i}\left(x-x_{i}\right)$, possibly with some repeated $x_{i}$, then $D$ is the sum of the points $\left(x_{i}, b\left(x_{i}\right)\right)$ on the curve. On the other hand, in our representation, we choose a line bundle $\mathcal{L}$ and represent $D$ by the global sections of $\mathcal{L}$ vanishing at $D$. We can take $\mathcal{L}=\mathcal{O}_{C}\left(N P_{\infty}\right)$, for $N \geq 3 g+1$, where $P_{\infty}$ is the "point at infinity" with $v_{P_{\infty}}(x)=2$ and $v_{P_{\infty}}(y)=2 g+1$. To simplify, we shall take $N=2 m$ to be even. Then a basis for $H^{0}(C, \mathcal{L})$, arranged in increasing order of pole at $P_{\infty}$, is $\left\{1, x, x^{2}, \ldots, x^{g}, y, x^{g+1}, x y, x^{g+2}, x^{2} y, \ldots, x^{m}\right\}$. Those sections that vanish at $D$ are then of the form $p(x) a(x)+q(x)(y-b(x))$, where $\operatorname{deg} p(x) \leq m-d$ and $\operatorname{deg} q(x) \leq m-1-g$. Our working with these sections using linear algebra can be thought of as performing polynomial operations on $a(x)$ and $b(x)$, but with a bound on the degree of intermediate results. (Of course, our algorithms also work in a more general setting.) An alternative way to deal with the above is to consider the affine curve $C-P_{0}$; its affine coordinate ring is 
$k[x, y] /\left(y^{2}-f(x)\right) \cong k[x] \oplus k[x] y$, and the elements vanishing at $D$ form an ideal generated (even as a $k[x]$ module) by the two elements $a(x)$ and $y-b(x)$.

\section{Embedding the Symmetric POWER of a CURVE into a Grassmannian}

Let $X$ be an algebraic variety over a field $k$; for the moment, we take $k$ to be algebraically closed. It is standard to try to embed $X$ into projective space by choosing a very ample line bundle $\mathcal{L}$ on $X$, and using $\mathcal{L}$ to get an embedding of $X$ into $\mathbf{P}\left(H^{0}(X, \mathcal{L})\right)$. Here $\mathbf{P}(V)$ is the projective space that parametrizes codimension one subspaces of $V$. The standard embedding associates $x \in X$ to the subspace $\left\{s \in H^{0}(X, \mathcal{L}) \mid s\right.$ vanishes at $\left.x\right\}$. More generally, one can try to embed $X$ into a Grassmannian variety, using a suitably positive rank $d$ vector bundle $\mathcal{E}$. We then associate to $x \in X$ the analogous subspace $W_{x}$ of global sections of $\mathcal{E}$ vanishing at $x$. Under suitable hypotheses, this gives us an embedding $\varphi: X \rightarrow \mathbf{G}_{d}(V)$, where $\mathbf{G}_{d}(V)$ parametrizes codimension $d$ subspaces $W$ of $V$. The map $\varphi$ can be composed with the Plücker embedding $\mathbf{G}_{d}(V) \rightarrow \mathbf{P}\left(\wedge^{d} V\right)$ to obtain an embedding of $X$ into projective space. For practical purposes, this may be unhelpful, as the dimension of

$\wedge \wedge^{d} V$ is $\left(\begin{array}{c}\operatorname{dim} V \\ d\end{array}\right)$, which is significantly greater than $\operatorname{dim} V$ once $d \geq 2$. If moreover $d$ increases with $\operatorname{dim} V$, the growth in the dimension can be exponential; for example, $\left(\begin{array}{c}2 n \\ n\end{array}\right)$ grows roughly as $4^{n} / \sqrt{\pi n}$ by Stirling's formula. This suggests that we would be better off working with subspaces of $V$, and doing explicit calculations using linear algebra, instead of passing to a possibly exponential number of explicit projective coordinates via the Plücker embedding. The case that interests us most is when $X$ is the $d$ th symmetric power $\operatorname{Sym}^{d}(C)$ of a curve $C$. We shall embed $\operatorname{Sym}^{d}(C)$ into a certain Grassmannian without explicitly mentioning vector bundles. The alert reader will see that we are essentially working with the Quot scheme by hand (see Section 8.2 of [BLR90]).

Let $g$ be the genus of $C$, take $d \geq 1$, and fix once and for all a line bundle $\mathcal{L}$ on $C$ of degree $N \geq 2 g+d \geq 2 g+1$. The Riemann-Roch theorem implies that the space $V=H^{0}(\mathcal{L})=H^{0}(C, \mathcal{L})$ has dimension $N+1-g$, and $\mathcal{L}$ gives an embedding of $C$ into $\mathbf{P}(V) \cong \mathbf{P}^{N-g}$. In fact, a well-known theorem of Castelnuovo, Mattuck, and Mumford states that this embedding of $C$ is projectively normal, and there is an even stronger theorem, due to Fujita and Saint-Donat, which states that if in fact $N \geq 2 g+2$, then the ideal of $C$ is generated by quadrics ([Laz89, Section 1.1). Now a point on $\operatorname{Sym}^{d}(C)$ can be identified with an effective divisor $D=\sum_{P} m_{P} P$ on $C$, of degree $d=\sum m_{P}$; we associate to $D$ the subspace $W_{D}$ given by

$$
W_{D}=H^{0}(\mathcal{L}-D) \subset V=H^{0}(\mathcal{L}) .
$$

Thus $W_{D}$ consists of the global sections $s$ of $\mathcal{L}$ vanishing at $D$ (i.e., $s$ vanishes at each $P$ to order at least $m_{P}$ ). Note that we are freely abusing notation by often failing to distinguish between line bundles and divisors, and by referring to tensor operations on line bundles additively when it suits us; it would be more standard to write $W_{D}=H^{0}(\mathcal{L}(-D))$. Due to the condition on the degree $N$, the line bundle $\mathcal{L}-D$ is nonspecial and has no base points, so $W_{D}$ has codimension $d$ in $V$, and we can recover $D$ from $W_{D}$. We summarize this discussion in the following lemma, while noting incidentally that the argument also extends to the trivial case $d=0$.

Lemma 2.1 (Representation of divisors). Let $C$ be an algebraic curve of genus $g$, and let $\mathcal{L}$ be a line bundle on $C$ of degree $N$. Let $D=\sum m_{P} P$ be an effective divisor on $C$ of degree $d$ (we allow $D$ to be empty, in which case $d=0$ ). Let 
$W_{D}=H^{0}(\mathcal{L}-D) \subset H^{0}(\mathcal{L})=V$. Then if $d \leq N-2 g+1$, it follows that $W_{D}$ has codimension $d$ in $V$. If furthermore $d \leq N-2 g$, then $H^{0}(\mathcal{L}-D)$ has no base points, in the sense that $H^{0}(\mathcal{L}-D-P) \subsetneq H^{0}(\mathcal{L}-D)$ for all points $P$ of $C$. In that case, the multiplicity $m_{P}$ can be recovered by noting that $m_{P}$ is the minimum order to which any section $s \in W_{D}$ vanishes at $P$ (viewing $s$ as a global section of $\mathcal{L})$.

Proof. This follows immediately from the Riemann-Roch theorem.

Thus the map $D \mapsto W_{D}$ is injective, and allows us to view $\operatorname{Sym}^{d}(C)$ as a subset of $\mathbf{G}_{d}(V)$. For $N$ large compared to $d$ and $g$, Theorem 8.2.8' of [BLR90] guarantees that this injection of points is an embedding of varieties; we suspect that this holds in our setting, but have not checked the matter further, as we do not need this fact. Another way to view this map is via the embedding of $C$ into $\mathbf{P}(V)$ given by our choice of $\mathcal{L}$. Given a degree $d$ divisor $D$ on $C$, then its points span a certain $d$-dimensional subspace $W_{D}^{*}$ of the dual space $V^{*}$ (now identifying points of $\mathbf{P}(V)$ with nonzero elements of $V^{*}$ up to multiplication of scalars). Assuming that the multiplicities in $D$ are all at most 1 (i.e., that $D=\sum_{i} P_{i}$ for distinct $P_{i}$ can be viewed as a reduced scheme), then $W_{D}^{*}$ is precisely the annihilator of $W_{D}$ with respect to the pairing between $V$ and $V^{*}$. If $D$ has some higher multiplicities, we can still make sense of this last statement by understanding $W_{D}^{*}$ to be spanned not only by $P$, but by the $m_{P}$ th infinitesimal neighborhood (i.e., osculating plane) of $P$ on $C$ (see GH94], pp. 248 and 264). We shall interchangeably use $W_{D}^{*}$ to refer to the $d$-dimensional subspace of $V^{*}$ and to the corresponding $(d-1)$-plane in $\mathbf{P}(V)$. In this point of view, the fact that $H^{0}(\mathcal{L}-D)$ has no base points means that the points of $D$ are linearly independent (even including multiplicity), and that the plane $W_{D}^{*}$ intersects $C$ precisely in the divisor $D$. This gives another way to see the injectivity of the map $D \mapsto W_{D}$.

So far, we have worked over an algebraically closed field. The above discussion continues to hold if $C$ and $\mathcal{L}$ are defined over an arbitrary perfect base field $k$, provided that we restrict ourselves to divisors $D$ rational over $k$. This means that for all Galois automorphisms $\sigma \in \operatorname{Gal}(\bar{k} / k)$, we have an equality of divisors (and not just of divisor classes) $\sigma D=D$. In that case, the vector spaces $V$ and $W_{D}$ are finite-dimensional over $k$, and all our work can be done over the field $k$. This is quite convenient computationally, as we do not need to work with individual points of $D$, which can themselves be defined over extensions of $k$. Still, our main goal in working with $\operatorname{Sym}^{d}(C)$ is to descend to the Jacobian $J$ of $C$. In that setting, the above notion of rationality can fall somewhat short: a point of $J(k)$ need not be representable by a divisor rational over $k$, since we now only require equality of the divisor classes $D$ and $\sigma(D)$. This problem arises only if $C$ itself has no points defined over $k$; in that case, the obstruction to lifting a point of $J(k)$ to a divisor rational over $k$ lies in the Brauer group of $k$ (see Remark 1.6 of [Mil86]). We are primarily interested in the case where $k$ is a finite field (so the Brauer group is trivial), so we shall henceforth ignore the above distinction between rationality questions on the symmetric product and on the Jacobian. We merely remark that in working with various spaces of sections of line bundles (such as $H^{0}(\mathcal{L}-D)$ ), we shall work over the algebraic closure $\bar{k}$ when needed, and then deduce the desired results for $k$. A typical example is the following lemma, which is a slight generalization of the theorem by Castelnuovo, Mattuck, and Mumford cited above. 
Lemma 2.2 ("Multiplication"). Let $C$ be a smooth, geometrically connected algebraic curve of genus $g$ over a perfect field $k$, and let $\mathcal{L}_{1}$ and $\mathcal{L}_{2}$ be two line bundles on $C$ (both defined over $k$ ), of degrees $d_{1}$ and $d_{2}$. Assume that $d_{1}, d_{2} \geq 2 g+1$. Then the canonical (multiplication) map

$$
\mu: H^{0}\left(\mathcal{L}_{1}\right) \otimes_{k} H^{0}\left(\mathcal{L}_{2}\right) \rightarrow H^{0}\left(\mathcal{L}_{1} \otimes \mathcal{L}_{2}\right)
$$

is surjective.

Proof. We sketch a proof, adapting the exposition in Sections 1.3 and 1.4 of [Laz89, which treats the case $\mathcal{L}_{1}=\mathcal{L}_{2}$. It is enough to prove the result after extending the base field from $k$ to $\bar{k}$, since base extension of linear maps defined over $k$ does not change the dimension of their kernels or images. This principle will be used constantly in this article.

We assume without loss of generality that $d_{1} \leq d_{2}$, and begin with the following exact sequence of vector bundles on $C$ (using the notation of Laz89]):

$$
0 \rightarrow \mathcal{M}_{\mathcal{L}_{1}} \rightarrow H^{0}\left(\mathcal{L}_{1}\right) \otimes \mathcal{O}_{C} \stackrel{\alpha}{\rightarrow} \mathcal{L}_{1} \rightarrow 0
$$

Here $\alpha$ is the "evaluation" map, and $\mathcal{M}_{\mathcal{L}_{1}}=\operatorname{ker} \alpha$. We have used the fact that $\mathcal{L}_{1}$ is base point free, i.e., that it is generated by global sections. Tensoring (2.3) with $\mathcal{L}_{2}$ and taking cohomology reduces our problem to showing that $H^{1}\left(\mathcal{M}_{\mathcal{L}_{1}} \otimes \mathcal{L}_{2}\right)=0$. To this end, choose $r=d_{1}-1-g$ points $P_{1}, \ldots, P_{r}$ on $C$ in general position (this is where we really need to work over $\bar{k}$ ). Then, by Lemma 1.4.1 and the beginning of the proof of Theorem 1.2.7 of [Laz89], we obtain the following exact sequence (where we have temporarily lapsed back into writing line bundles multiplicatively) 1

$$
0 \rightarrow \mathcal{L}_{1}^{-1}\left(\sum_{i=1}^{r} P_{i}\right) \rightarrow \mathcal{M}_{\mathcal{L}_{1}} \rightarrow \bigoplus_{i=1}^{r} \mathcal{O}_{C}\left(-P_{i}\right) \rightarrow 0
$$

Tensoring this sequence with $\mathcal{L}_{2}$ and taking cohomology reduces our problem to showing, first, that $H^{1}\left(\mathcal{L}_{2}\left(-P_{i}\right)\right)=0$ for all $i$ (as is clear by using Serre duality and looking at degrees), and, second, that $H^{1}\left(\mathcal{L}_{2} \otimes \mathcal{L}_{1}^{-1}\left(\sum_{i=1}^{r} P_{i}\right)\right)=0$. This last statement is equivalent to requiring $H^{0}\left(\mathcal{K} \otimes \mathcal{L}_{2}^{-1} \otimes \mathcal{L}_{1}\left(-\sum_{i=1}^{r} P_{i}\right)\right)=0$, where we write $\mathcal{K}$ for the canonical bundle on $C$. Since we have assumed that $d_{2} \geq$ $d_{1}$, we see that the line bundle $\mathcal{L}_{3}=\mathcal{K} \otimes \mathcal{L}_{2}^{-1} \otimes \mathcal{L}_{1}$ has degree at most $2 g-2$. Therefore $\operatorname{dim} H^{0}\left(\mathcal{L}_{3}\right) \leq g$, because we can embed $H^{0}\left(\mathcal{L}_{3}\right)$ into the $g$-dimensional space $H^{0}\left(\mathcal{L}_{3}(D)\right)$, where $D$ is any effective divisor of degree $2 g-1-\operatorname{deg} \mathcal{L}_{3}$. Since the $r$ points $P_{1}, \ldots, P_{r}$ impose $r \geq g$ zeros, we obtain that $H^{0}\left(\mathcal{L}_{3}\left(-\sum_{i=1}^{r} P_{i}\right)\right)=0$ for generic $P_{1}, \ldots, P_{r}$, as desired.

Our last result in this section is a converse of sorts to Lemma 2.2 since it describes a kind of "division" of sections of line bundles. This relatively easy lemma/algorithm is crucial to our results, perhaps even more so than the somewhat deeper Lemma 2.2

\footnotetext{
${ }^{1}$ This is where we use the fact that $d_{1} \geq 2 g+1$, so $r \geq g$, and we can guarantee that $\mathcal{L}_{1}\left(-\sum P_{i}\right)$ not only has a two-dimensional space of global sections, but is also base point free for a generic choice of points $P_{1}, \ldots P_{r}$, because it is a generic line bundle of degree $g+1$. We then obtain (2.4) by combining (2.3) with an analogous exact sequence for $\mathcal{L}_{1}\left(-\sum P_{i}\right)$, and using the snake lemma.

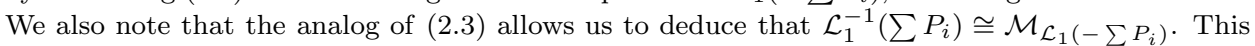
is known as the "base point free pencil trick"; for more details, refer to the exposition in [Laz89] and to ACGH85], pp. 126 and 151, particularly Exercise K.
} 
Lemma/Algorithm 2.3 ("Division"). Let $C$ be a curve as in Lemma 2.2. Let $\mathcal{L}_{1}$ and $\mathcal{L}_{2}$ be line bundles (written multiplicatively in this lemma/algorithm), and let $D_{1}$ and $D_{2}$ be effective divisors on $C$, where everything is defined over $k$. (We allow $D_{1}$ or $D_{2}$ to be zero.) Assume that $\mathcal{L}_{2}\left(-D_{2}\right)$ has no base points (over $\bar{k}$ ); for example, it is sufficient to have $\operatorname{deg} \mathcal{L}_{2}-\operatorname{deg} D_{2} \geq 2 g$. Let $\mu: H^{0}\left(\mathcal{L}_{1}\right) \otimes$ $H^{0}\left(\mathcal{L}_{2}\right) \rightarrow H^{0}\left(\mathcal{L}_{1} \otimes \mathcal{L}_{2}\right)$ be the multiplication map, as before. Assume that we know the subspaces $H^{0}\left(\left(\mathcal{L}_{1} \otimes \mathcal{L}_{2}\right)\left(-D_{1}-D_{2}\right)\right) \subset H^{0}\left(\mathcal{L}_{1} \otimes \mathcal{L}_{2}\right)$, and $H^{0}\left(\mathcal{L}_{2}\left(-D_{2}\right)\right) \subset$ $H^{0}\left(\mathcal{L}_{2}\right)$. Then we can compute $H^{0}\left(\mathcal{L}_{1}\left(-D_{1}\right)\right)$ to be the space

$(2.5)\left\{s \in H^{0}\left(\mathcal{L}_{1}\right) \mid \forall t \in H^{0}\left(\mathcal{L}_{2}\left(-D_{2}\right)\right), \quad \mu(s \otimes t) \in H^{0}\left(\left(\mathcal{L}_{1} \otimes \mathcal{L}_{2}\right)\left(-D_{1}-D_{2}\right)\right)\right\}$.

Note that for computational purposes, it is enough to let $t$ range only over a basis for $H^{0}\left(\mathcal{L}_{2}\left(-D_{2}\right)\right.$ ) (and not over the whole space) in equation (2.5).

Proof. The fact that $H^{0}\left(\mathcal{L}_{1}\left(-D_{1}\right)\right)$ is a subset of the space defined in (2.5) is immediate. As for the reverse inclusion, it follows from the fact that $H^{0}\left(\mathcal{L}_{2}\left(-D_{2}\right)\right)$ has no base points. To see why, extend scalars to $\bar{k}$, and write $D_{1}=\sum_{P} m_{P} P$ and $D_{2}=\sum_{P} n_{P} P$, where only finitely many of the $m_{P}$ and $n_{P}$ are nonzero. For any point $P$ on $C$, base point freeness means that we can find $t \in H^{0}\left(\mathcal{L}_{2}\left(-D_{2}\right)\right)$ which (when viewed as a section of $H^{0}\left(\mathcal{L}_{2}\right)$ ) vanishes at $P$ to order exactly $n_{P}$. Thus, if $s \in H^{0}\left(\mathcal{L}_{1}\right)$ satisfies the condition in equation (2.5), then $\mu(s \otimes t)$ vanishes at $P$ to order at least $m_{P}+n_{P}$, from which we conclude that $s$ vanishes at $P$ to order at least $m_{P}$. Since $P$ was arbitrary, we obtain that $s \in H^{0}\left(\mathcal{L}_{1}\left(-D_{1}\right)\right)$, as desired.

Remark 2.4. Lemma/Algorithm 2.3 works even if the output, $H^{0}\left(\mathcal{L}_{1}\left(-D_{1}\right)\right)$, fails to be base point free. The fact that our section $s$ is required to vanish at $D_{1}$ may well have severe consequences for the behavior of $s$ at other points of $C$.

Remark 2.5. In Remark 3.8 below, we shall see that calculating $H^{0}\left(\mathcal{L}_{1} \otimes \mathcal{L}_{2}\right)$ as the image of $\mu$, as in Lemma 2.2, can be done in $O\left(\delta^{4}\right)$ steps, if the dimensions of the vector spaces involved are of the order of $\delta$. (In our applications, $\delta$ is a small constant times $g$.) We caution the reader to avoid an overly naive implementation of the calculation that would take time $O\left(\delta^{5}\right)$. We shall also see in the same remark below that the operation in Lemma/Algorithm 2.3 can be implemented in time $O\left(\delta^{4}\right)$.

\section{BASIC ALGORITHMS ON DIVISORS}

As in Section 2, let $C$ be a curve of genus $g$ over a perfect field $k$, and fix a line bundle $\mathcal{L}$ on $C$ of large degree $N$, which will be at least $2 g+1$, and typically somewhat larger. Throughout this section, except briefly in Theorem/Algorithm 3.14 the letters $D, D^{\prime}$, and $E$ will refer to effective divisors on $C$ (that are rational over $k$ ), of degrees $d, d^{\prime}$, and $e$.

We represent an effective divisor $D$ of degree $d \leq N-2 g$ by the codimension $d$ subspace $W_{D}=H^{0}(\mathcal{L}-D)$ of the vector space $V=H^{0}(\mathcal{L})$; alternatively, we can work with its annihilator $W_{D}^{*} \subset V^{*}$, corresponding to the span of the points of $D$ (counting multiplicity) in the projective embedding of $C$ into $\mathbf{P}(V)$. In later sections, we give various choices of parameters that allow us to apply the algorithms of this section to compute in the Jacobian of $C$. For ease of following the exposition in this section, we advise the reader to keep in mind the application given in Section 4 In that setting, $g \geq 1$, and $\mathcal{L}$ will have the form $3 \mathcal{L}_{0}=\mathcal{O}_{C}\left(3 D_{0}\right)$ for $D_{0}$ a divisor of degree $d_{0} \geq 2 g$; there is some benefit in even assuming that $d_{0} \geq 2 g+1$, as this 
allows us to use either Theorem/Algorithm 3.6 or 3.13 whereas if we use $d_{0}=2 g$, we can only use the latter Theorem/Algorithm. The reader should probably use the value $d_{0}=2 g+1$, whence $N=3 d_{0}=6 g+3$. The values of $d$ that will appear in our application are $d=d_{0}\left(=2 g+1\right.$, say) and $d=2 d_{0}(=4 g+2)$; an effective divisor $D$ of degree $d_{0}$ will be called small, and a divisor of degree $2 d_{0}$ will be called large. A small divisor $D$ will describe a point on the Jacobian $J$ corresponding to the line bundle $\mathcal{O}_{C}\left(D-D_{0}\right)$, and a large divisor $D^{\prime}$ will usually arise as an effective divisor equivalent to $3 D_{0}-D$ for some small divisor $D$. Nonetheless, we carry through our analysis for more general values of $N$ and $d$, subject to certain inequalities. This allows for different versions of our algorithms, depending on which parameters and design choices are most suitable in a given context.

We begin by identifying the subspaces $W_{D} \cap W_{E}$ and $W_{D}+W_{E}$, in terms of divisors related to $D$ and $E$. These subspaces are dual to $W_{D}^{*}+W_{E}^{*}$ and $W_{D}^{*} \cap W_{E}^{*}$. From either point of view (either thinking of $W_{D}$ as a space of sections which vanish at $D$, or of $W_{D}^{*}$ as the span of the points of $D$ ), it is very plausible that these new subspaces should correspond to the divisors $D \cup E$ and $D \cap E$, at least if all the multiplicities of points are at most 1. The content of Proposition/Algorithm 3.2 is that a result of this kind holds in general, provided we make the following definition.

Definition 3.1. If $D=\sum m_{P} P$ and $E=\sum n_{P} P$ are two (effective) divisors, then we define

$$
D \cup E=\sum \max \left(m_{P}, n_{P}\right) P, \quad D \cap E=\sum \min \left(m_{P}, n_{P}\right) P .
$$

Note that if $D$ and $E$ are both defined over $k$, then so are $D \cup E$ and $D \cap E$.

Proposition/Algorithm 3.2 (Union and intersection). Given $D$ and $E$ as in Definition 3.1, then in all cases $W_{D} \cap W_{E}=W_{D \cup E}$ (or, dually, $\left.W_{D}^{*}+W_{E}^{*}=W_{D \cup E}^{*}\right)$. If furthermore $\operatorname{deg}(D \cup E) \leq N-2 g$, then we can recover $D \cup E$ from the subspace $W_{D \cup E}$, and we also obtain $W_{D}+W_{E}=W_{D \cap E}$ (dually, $W_{D}^{*} \cap W_{E}^{*}=W_{D \cap E}^{*}$ ).

Proof. The first statement is trivial, as we are requiring sections to vanish at $P$ simultaneously to order at least $m_{P}$ and at least $n_{P}$. The condition on the degree of $D \cup E$ is to ensure that $H^{0}(\mathcal{L}-(D \cup E))$ is base point free by Lemma 2.1 so that we can recover $D \cup E$. Moreover, $\operatorname{codim} W_{D \cup E}=\operatorname{deg}(D \cup E)$ in that case. As for $W_{D \cap E}$, we always have $W_{D}+W_{E} \subset W_{D \cap E}$. If we know that $\operatorname{deg}(D \cup E) \leq N-2 g$, then we can conclude equality by comparing dimensions, since $\operatorname{codim}\left(W_{D}+W_{E}\right)=$ $\operatorname{codim} W_{D}+\operatorname{codim} W_{E}-\operatorname{codim}\left(W_{D} \cap W_{E}\right)$, and an analogous equality holds for the degrees of the corresponding divisors.

Remark 3.3. In the context of Section 4, Proposition/Algorithm 3.2 allows us to find the union and intersection of two small divisors $D$ and $E$, since in that case the degree of $D \cup E$ cannot exceed $d+e=2 d_{0}=N-d_{0} \leq N-2 g$. In case these divisors are disjoint, as can be measured by looking at their intersection, this gives a simple way to compute the sum of these divisors. We formalize this below, and throw in an easy algorithm to see if one divisor is contained in the other. By this we mean the following definition:

$$
D=\sum m_{P} P \subset E=\sum n_{P} P \Longleftrightarrow \text { for all } P, m_{P} \leq n_{P} .
$$

Proposition/Algorithm 3.4 (Disjointness and inclusion). Given divisors $D$ and $E$, of degrees $d$ and e respectively, then: 
(1) If $d+e \leq N-2 g$, then we can test if $D \cap E=\emptyset$ by checking if $W_{D}+W_{E}=V$ (alternatively, $W_{D}^{*} \cap W_{E}^{*}=0$ ). In that case, $D+E=D \cup E$ can be calculated as $W_{D+E}=W_{D} \cap W_{E}$, by Proposition/Algorithm 3.2. An alternative to checking if $W_{D}+W_{E}=V$ is to simply compute $W_{D} \cap W_{E}$ and to see if the intersection has codimension $d+e$.

(2) If $d, e \leq N-2 g$, then we can test if $D \subset E$ (in the sense of (3.2)) by testing whether $W_{E} \subset W_{D}$ (dually, if $W_{D}^{*} \subset W_{E}^{*}$ ).

Remark 3.5. The linear algebra calculations to which we reduce our problem in our algorithms can be tested easily using standard techniques of Gaussian elimination. This involves choosing a basis for $V$ (hence an isomorphism $V \cong k^{N+1-g}$ ), and expressing everything in terms of the resulting coordinates on $V$. A subspace such as $W_{D}$ can then be represented in terms of a basis for $W_{D}$, expressed in terms of the coordinates on $V$. Alternatively, one can work with $W_{D}^{*}$ instead of $W_{D}$, since for small divisors, $W_{D}^{*}$ has smaller dimension. The drawback to this is that using $W_{D}^{*}$ appears to be less efficient for our later algorithms, which involve the multiplication map $\mu$. We also refer the reader to a side observation in Remark 3.8 for an idea of how to test whether $D$ and $E$ are disjoint even if $d+e>N-2 g$; however, that method requires the slightly stronger hypothesis that $d, e \leq N-2 g-1$. Alternatively, one can replace $\mathcal{L}$ with a line bundle of higher degree, as in the discussion at the end of Section 5 .

We now describe an algorithm to add two divisors in general, whether or not they are disjoint. We need to assume knowledge of the multiplication map

$$
\mu: V \otimes V=H^{0}(\mathcal{L}) \otimes_{k} H^{0}(\mathcal{L}) \rightarrow H^{0}(2 \mathcal{L}) .
$$

In the case that interests us, $N \geq 2 g+2$. Then knowledge of $\mu$ is tantamount to knowing the quadratic polynomials that define $C$ in the projective embedding given by $\mathcal{L}$, as mentioned just before equation (2.1). The map $\mu$ can be represented explicitly in terms of bases for $V$ and for $H^{0}(2 \mathcal{L})$. The technique in the theorem below is fundamental, and plays a role in all the remaining algorithms of this article. We give a geometric interpretation of this technique after the proof of Proposition/Algorithm 3.9 below. In Theorem/Algorithm 3.13 below, we give a second algorithm for addition of divisors, using some similar ideas. The other algorithm works in a slightly more general context than the following algorithm, but the first algorithm is perhaps simpler to explain.

Theorem/Algorithm 3.6 (Addition of divisors (first method)). Let $D$ and $E$ satisfy $d, e \leq N-2 g-1$. Then we can compute $W_{D+E}$ in the following steps:

(1) Restricting $\mu$ to the subspace $W_{D} \otimes W_{E}=H^{0}(\mathcal{L}-D) \otimes H^{0}(\mathcal{L}-E)$ of $V \otimes V$, we obtain from Lemma 2.2 that $\mu\left(W_{D} \otimes W_{E}\right)=H^{0}(2 \mathcal{L}-D-E)$. The subspace $H^{0}(2 \mathcal{L}-D-E)$ of $H^{0}(2 \mathcal{L})$ can be computed as the span of $\mu\left(s_{i} \otimes s_{j}^{\prime}\right)$, as $s_{i}$ and $s_{j}^{\prime}$ range over bases for $W_{D}$ and $W_{E}$ respectively.

(2) Using Lemma/Algorithm 2.3, we compute

$$
W_{D+E}=\left\{s \in V \mid \forall t \in V, \quad \mu(s \otimes t) \in H^{0}(2 \mathcal{L}-D-E)\right\} .
$$

Recall that one only needs to let $t$ range over a basis for $V$.

Proof. In order to apply Lemma/Algorithm [2.3, we need to observe that $H^{0}(\mathcal{L})$ is base point free. This holds by Lemma 2.1 since our assumptions imply that $N \geq 2 g+1+d \geq 2 g$. 
Remark 3.7. In order for knowledge of $W_{D+E}$ to allow us to uniquely recover $D+E$, we need to know that $d+e \leq N-2 g$; in the setting of Section 4 , for example, this would require both $D$ and $E$ to be small (and $d_{0} \geq 2 g+1$ ), even though the above algorithm correctly calculates $W_{D+E}$ in case one of the divisors is small, and the other large. Nonetheless, for use in Theorems/Algorithms 4.1 and 5.5 and elsewhere, we have presented the above algorithm in the general case, even if $W_{D+E}$ does not uniquely determine $D+E$. We note as an aside that this last case happens precisely when $H^{0}(\mathcal{L}-D-E)$ has base points, and in that case the inclusion

$$
\mu\left(H^{0}(\mathcal{L}-D-E) \otimes H^{0}(\mathcal{L})\right) \subset H^{0}(2 \mathcal{L}-D-E)
$$

will be strict, since $H^{0}(2 \mathcal{L}-D-E)$ will never have base points, by Lemma 2.1 (This of course does not affect the validity of our proof; in the easy half of the proof of Lemma/Algorithm 2.3, we only need the inclusion in equation (3.5) to obtain that $W_{D+E} \subset\{s$ as above $\}$.)

Remark 3.8. We discuss some practical issues in implementing the above algorithm, beyond the fact that Theorem/Algorithm [3.6] settles the theoretical issue of computing $W_{D+E}$. We also discuss the running time of the algorithm. Let the dimensions of the vector spaces involved in the calculation be of the order of $\delta$ (this is approximately $\operatorname{dim} V=N+1-g$, which is $O(g)$ for all our applications). Then an overly naive implementation of the algorithm as stated above takes time $O\left(\delta^{5}\right)$, but a little care brings this down to $O\left(\delta^{4}\right)$. We can use some randomness to make the algorithm slightly faster, but still taking $O\left(\delta^{4}\right)$ steps. (We remind the reader that we count an operation in the field $k$ as a single step, taking one unit of time.) We note that our second algorithm below, Theorem/Algorithm 3.13 also takes time $O\left(\delta^{4}\right)$.

We first discuss why the naive implementation of Theorem/Algorithm3.6 runs in time $O\left(\delta^{5}\right)$, and how to reduce this time to $O\left(\delta^{4}\right)$. We assume that $\mu$ is implemented in terms of fixing a basis $\left\{t_{i}\right\}$ for $V$, and storing all the products $v_{i j}=\mu\left(t_{i} \otimes t_{j}\right) \in$ $H^{0}(2 \mathcal{L})$. Moreover, a subspace $W_{D}$ is represented by choosing a basis, and writing all the basis elements in terms of the $t_{i}$. Then it takes $O\left(\delta^{3}\right)$ operations to calculate each individual $\mu\left(s_{i} \otimes s_{j}^{\prime}\right)$, as $s_{i}$ and $s_{j}^{\prime}$ range over bases for $W_{D}$ and $W_{E}$. Since there are $O\left(\delta^{2}\right)$ such pairs $\left(s_{i}, s_{j}^{\prime}\right)$, it seems that we will need $O\left(\delta^{5}\right)$ steps merely to write down a spanning set for $H^{0}(2 \mathcal{L}-D-E)$, viewed as a subspace of the $O(\delta)$ dimensional space $H^{0}(2 \mathcal{L})$. However, we can proceed more quickly, at the expense of using more memory, as follows: First calculate (and store) each product $w_{i j}=$ $\mu\left(s_{i} \otimes t_{j}\right)$, as $s_{i}$ ranges over a basis for $W_{D}$, and $t_{j}$ ranges over the standard basis for $V$. This takes only $O\left(\delta^{4}\right)$ steps, since each individual multiplication $\mu\left(s_{i} \otimes t_{j}\right)$ now needs only $O\left(\delta^{2}\right)$ operations, as it involves only the $v_{i^{\prime} j}$ for a fixed $j$ and varying $i^{\prime}$. Now if $E=0$, we are already done; more generally, we use the $w_{i j}$ to assemble all the products $\mu\left(s_{i} \otimes s_{j}^{\prime}\right)$ in a further $O\left(\delta^{4}\right)$ steps. Since these products will virtually always be linearly dependent, we should then use Gaussian elimination to reduce these products to a basis for $H^{0}(2 \mathcal{L}-D-E)$. This takes $O\left(\delta^{4}\right)$ operations, since we have $O\left(\delta^{2}\right)$ vectors to reduce inside the $O(\delta)$-dimensional space $H^{0}(2 \mathcal{L}) .2$

\footnotetext{
${ }^{2}$ It would be interesting to try to use the fact that for fixed $i$ and varying $j$ (or vice versa), the products $\mu\left(s_{i} \otimes s_{j}^{\prime}\right)$ are known to be linearly independent before we start Gaussian elimination. Still, there are advantages to obtaining a basis for $H^{0}(2 \mathcal{L}-D-E)$ in echelon form, once we invoke Lemma/Algorithm 2.3
} 
All of this takes some time. So in practice (at least when $d+e \leq N-2 g$ ), one should rely on the fact that the divisors $D$ and $E$ are likely to be disjoint, and simply compute $W_{D} \cap W_{E}$, as mentioned in Proposition/Algorithm 3.4. This only takes $O\left(\delta^{3}\right)$ operations. If the intersection has the right codimension $d+e$, which should happen rather often, then we immediately obtain $W_{D+E}$ as this intersection. We note that in the undesirable case $d+e>N-2 g$, it does not seem to be worth the computational effort to check if $D$ and $E$ are disjoint; one might as well use Theorem/Algorithm 3.6 directly. (Here is one idea of how one can adapt our algorithms to check that $D$ and $E$ are disjoint, even if $d+e>N-2 g$ : First calculate $H^{0}(2 \mathcal{L}-D)=\mu\left(W_{D} \otimes V\right)$, and similarly $H^{0}(2 \mathcal{L}-E)$; this works, and takes time $O\left(\delta^{4}\right)$ as before. Then find the intersection $H^{0}(2 \mathcal{L}-D) \cap H^{0}(2 \mathcal{L}-E)$ inside the larger ambient space $H^{0}(2 \mathcal{L})$ instead of $H^{0}(\mathcal{L})$. This amounts to using $2 N$ instead of $N$, which allows us to proceed as in Proposition/Algorithm 3.4 since $d+e$ is now less than $2 N-2 g$.)

Coming back to the issue of calculating $\mu\left(W_{D} \otimes W_{E}\right)$, we note that we can also try to avoid calculating all $O\left(\delta^{2}\right)$ products $\mu\left(s_{i} \otimes s_{j}^{\prime}\right)$. Instead, we randomly choose $O(\delta)$ pairs of the form $\left(s, s^{\prime}\right)$, where $s \in W_{D}$ and $s^{\prime} \in W_{E}$ are random (nonzero) elements. In that case, the resulting products $\mu\left(s \otimes s^{\prime}\right)$ are likely to already span $H^{0}(2 \mathcal{L}-D-E)$, especially if we take, say, twice as many pairs $\left(s, s^{\prime}\right)$ as the dimension of $H^{0}(2 \mathcal{L}-D-E)$. With high probability, this reduces the time we spend on finding $\mu\left(W_{D} \otimes W_{E}\right)$, but it still involves $O\left(\delta^{4}\right)$ operations, since one must calculate the $\mu\left(s \otimes s^{\prime}\right)$. On the other hand, this reduces the time spent on Gaussian elimination to $O\left(\delta^{3}\right)$, but without an assurance of success, as we have not necessarily used a full spanning set for $H^{0}(2 \mathcal{L}-D-E)$.

As for the "division" calculation in step 2 of Theorem/Algorithm 3.6, it can be done in $O\left(\delta^{4}\right)$ operations as follows: First, find a matrix $M$, representing a linear transformation from $H^{0}(2 \mathcal{L})$ to $k^{d+e}$, whose kernel is precisely $H^{0}(2 \mathcal{L}-D-E)$. This amounts to finding a basis of the annihilator $H^{0}(2 \mathcal{L}-D-E)^{*} \subset H^{0}(2 \mathcal{L})^{*}$, and can be done in time at most $O\left(\delta^{3}\right)$ (even $O\left(\delta^{2}\right)$, if the basis for $H^{0}(2 \mathcal{L}-D-E)$ is already in echelon form). Recall that we have on hand all the products $v_{i j}=$ $\mu\left(t_{i} \otimes t_{j}\right) \in H^{0}(2 \mathcal{L})$, where the $t_{i}$ are a basis for $V$. We now need to solve for $s=\sum_{i} x_{i} t_{i}$ such that for all $j, \sum_{i} x_{i} M\left(v_{i j}\right)=(0, \ldots, 0) \in k^{d+e}$. Computing the various $M\left(v_{i j}\right)$ takes $O\left(\delta^{4}\right)$ steps, and the resulting Gaussian elimination to solve for the tuples $\left(\ldots, x_{i}, \ldots\right)$ representing $s$ takes $O\left(\delta^{4}\right)$ operations, being a system of $O\left(\delta^{2}\right)$ equations in $O(\delta)$ variables. We point out that we will later still be able to perform similar calculations (inspired by Lemma/Algorithm 2.3) in $O\left(\delta^{4}\right)$ operations, even if we no longer have $t$ ranging over all of $V$. This is necessary for equation (3.7) in Proposition/Algorithm 3.9. as well as Theorems/Algorithms 3.10. 3.13, and 3.14 The idea is that we need to compute $v_{i j}^{\prime}=\mu\left(t_{i} \otimes t_{j}^{\prime}\right)$ for $t_{i}$ as before, but with $t_{j}^{\prime}$ ranging over a basis for some suitable subspace of $V$. Each $v_{i j}^{\prime}$ can be computed from the existing collection of $v_{i j}$ in $O\left(\delta^{2}\right)$ operations, so it takes time $O\left(\delta^{4}\right)$ to assemble the $v_{i j}^{\prime}$, and the calculation proceeds as before.

We next give another illustration of the ideas in Theorem/Algorithm 3.6 Given two divisors $D=\sum m_{P} P$ and $E=\sum n_{P} P$, we may wish to calculate the divisor

$$
E \backslash D=\sum \max \left(0, n_{P}-m_{P}\right) P \text {. }
$$

If the multiplicities are at most 1 , then this is the usual operation on sets of points. If $D \subset E$, this is just subtraction of divisors. 
Proposition/Algorithm 3.9 (Set subtraction). Given $D$ and $E$, with $d \leq N-2 g$ and $e \leq N-2 g-1$, we can calculate $W_{E \backslash D}$ as follows: First, calculate $H^{0}(2 \mathcal{L}-E)=$ $\mu\left(W_{E} \otimes V\right)$. Then compute

$$
W_{E \backslash D}=\left\{s \in V \mid \forall t \in W_{D}, \quad \mu(s \otimes t) \in H^{0}(2 \mathcal{L}-E)\right\} .
$$

Proof. Lemma 2.2 gives us the surjectivity of $\mu: H^{0}(\mathcal{L}-E) \otimes H^{0}(\mathcal{L}) \rightarrow H^{0}(2 \mathcal{L}-E)$. As for (3.7), it follows from a modification of the argument in Lemma/Algorithm2.3, since $H^{0}(\mathcal{L}-D)$ has no base points. Note again that the computation on (3.7) can be done effectively by letting $t$ range only over a basis for $W_{D}$.

We now come to the most important basic algorithm in this section. It is close to Theorem/Algorithm 3.6 and Proposition/Algorithm 3.9, but we shall first pause to describe what the algorithm does geometrically. Our discussion will also shed light on Theorem/Algorithm 3.6. The geometric picture is best understood in terms of the projective embedding of $C$ into $\mathbf{P}(V)$ given by $\mathcal{L}$, and in terms of ideals of the graded projective coordinate ring of $\mathbf{P}(V)$. To this end, let $\mathcal{A}=\operatorname{Sym}^{*} V$ be this projective coordinate ring; $\mathcal{A}$ is the symmetric algebra on $V$, and an element of $V$ is a linear (degree one) polynomial whose vanishing set defines a hyperplane in $\mathbf{P}(V)$. The curve $C$ is defined by a homogeneous ideal $\mathcal{I}_{C}$, which is generated in our application by elements of degree two, and these elements can more or less be identified with the kernel of $\mu: V \otimes V \rightarrow H^{0}(2 \mathcal{L})$. (The quotient $\mathcal{A} / \mathcal{I}_{C}$ is the projective coordinate $\operatorname{ring}$ of $C$, and $\mu$ is just multiplication in this quotient.) Given a divisor $D$ and its associated subspaces $W_{D}$ and $W_{D}^{*}$, we shall abuse notation and also write $W_{D}^{*}$ for the corresponding plane in $\mathbf{P}(V)$, as described just after Lemma 2.1. In that case, we write $\mathcal{I}_{W}$ for the ideal defining this plane; $\mathcal{I}_{W}$ is then generated by $W_{D}$, which consists of the linear functions vanishing on $W_{D}^{*}$. As we have seen before, $D=W_{D}^{*} \cap C$. In terms of ideals, this means that $D$ is defined by the ideal $\mathcal{I}_{D}=\mathcal{I}_{W}+\mathcal{I}_{C}=W_{D} \mathcal{A}+\mathcal{I}_{C}$.

Now our next algorithm implements the following operation: Given a section $f \in W_{D}$, we know that $f$ (viewed as a section of $\mathcal{L}$ ) vanishes at a divisor of the form $E=D+D^{\prime}$ belonging to the equivalence class of $\mathcal{L}$. We then wish to find $W_{D^{\prime}}$, essentially by the same method as in Proposition/Algorithm 3.9 Geometrically, the zero set of $f$ is a hyperplane $H$ passing through $D$, and its ideal $\mathcal{I}_{H}$ is generated by $f$. Then the divisor $E$ is the intersection $H \cap C$, and is defined by $\mathcal{I}_{E}=\mathcal{I}_{H}+\mathcal{I}_{C}=f \mathcal{A}+\mathcal{I}_{C}$. As a zero-dimensional scheme, we then obtain that $D^{\prime}=E \backslash D$ should be defined by

$$
\mathcal{I}_{D^{\prime}}=\left(\mathcal{I}_{E}: \mathcal{I}_{D}\right)=\left\{a \in \mathcal{A} \mid a \mathcal{I}_{D} \subset \mathcal{I}_{E}\right\}=\left\{a \in \mathcal{A} \mid a W_{D} \subset f \mathcal{A}+\mathcal{I}_{C}\right\} .
$$

Namely, functions should vanish on $D^{\prime}$ if and only if when we multiply them by a function vanishing on $D$, we obtain a function vanishing on $E=D \cap D^{\prime}=H \cap C$. This fact is not so immediate if $D$ and $E$ have multiplicities greater than 1 ; it is essential, for example, that our divisors lie on a fixed curve, so that every extra order of vanishing at a point $P$ imposes exactly one more condition on homogeneous polynomial functions, provided that the degree of $E$ is not too large. At any rate, we are not interested in the ideal defining $D^{\prime}$ itself, but rather in the plane $W_{D^{\prime}}^{*}$ spanned by $D^{\prime}$. Dually, we only wish to determine $W_{D^{\prime}}$, which consists of the linear functions vanishing on $D^{\prime}$. Thus we only seem to need to find the degree one elements of $\mathcal{I}_{D^{\prime}}$. Using (3.8), this leads us to the calculation that we present below in Theorem/Algorithm[3.10. (Multiplication of linear functions and taking 
the quotient by the quadratic elements of $\mathcal{I}_{C}$ is exactly the map $\mu$.) Again, the fact that we can content ourselves with the degree one elements of $\mathcal{I}_{D^{\prime}}$ is not so trivial: in general, the vanishing set of a homogeneous ideal on projective space depends only on the saturation of that ideal (exercise II.5.10 of [Har77); note that step 2 in Theorem/Algorithm 3.6 is effectively computing a saturation). Now knowing the degree one elements of a saturation can in principle involve elements of arbitrarily high degree in the ideal. To do this algorithmically in general would probably involve Gröbner basis techniques, which will typically be slower than our methods the Gröbner basis calculations can take exponential time in the worst case (although they are efficient for "most" calculations), and even their average case behavior is likely to be slower than our linear algebra calculations. The point of our techniques is that we can use them to justify the geometric reasoning in the above heuristic argument. We therefore (finally) present the following result.

Theorem/Algorithm 3.10 (Flipping algorithm). Let $D$ be an effective divisor of degree $d \leq N-2 g$. Take a nonzero element $f \in W_{D}$, and write the divisor of $f$ (viewed as a section of $\mathcal{L}$ ) as

$$
(f)=E=D+D^{\prime}
$$

Thus $d^{\prime}=\operatorname{deg} D^{\prime}=N-d$, and $D^{\prime}$ belongs to the divisor class of $\mathcal{L}-D$. Then we can compute $W_{D^{\prime}}$ in the following steps:

(1) Calculate $H^{0}\left(2 \mathcal{L}-D-D^{\prime}\right)=\mu\left(f \otimes H^{0}(\mathcal{L})\right)$.

(2) Compute $W_{D^{\prime}}=\left\{s \in H^{0}(\mathcal{L}) \mid\right.$ for all $t$ in (a basis for) $W_{D}, \mu(s \otimes t) \in$ $\left.H^{0}\left(2 \mathcal{L}-D-D^{\prime}\right)\right\}$.

Note that if furthermore $d \geq 2 g$, then knowledge of $W_{D^{\prime}}$ determines $D^{\prime}$ uniquely, by Lemma 2.1.

Proof. The identity in the first step (which amounts to computing the image of the degree two elements of $f \mathcal{A}$ in the quotient $\mathcal{A} / \mathcal{I}_{C}$ ) does not need Lemma 2.2 but turns out to be much simpler. In fact, multiplication by $f$ is a bijection between $H^{0}(\mathcal{L})$ and $H^{0}\left(2 \mathcal{L}-D-D^{\prime}\right)$, the inverse being division by $f$ (which does not introduce any poles!). Then the second step above (which is the degree one part of the left-hand side of (3.8), whose computation involves some degree two elements on the right-hand side) follows from Lemma/Algorithm 2.3 since $H^{0}(\mathcal{L}-D)$ has no base points.

Remark 3.11. In the setting of our application in Section! we have $\mathcal{L}=\mathcal{O}_{C}\left(3 D_{0}\right)$, $N=3 d_{0}$, and $d_{0} \geq 2 g$. The above algorithm then allows us to go from a divisor $D$ (which may be small or large, corresponding to $d=d_{0}$ or $2 d_{0}$ respectively) to a complementary divisor $D^{\prime}$ (which is then respectively large or small), such that $D+D^{\prime}$ is linearly equivalent to $3 D_{0}$. This is reminiscent of the theory behind the Weierstrass embedding of an elliptic curve via a cubic equation, except that we are contenting ourselves with quadratic equations and must therefore use a more positive line bundle for our embedding of $C$. In Section [5, we go through analogs of our construction using smaller divisors (e.g., $d_{0}=g+1$, and $N=\operatorname{deg} \mathcal{L}=3 g+3$ ), working instead with "higher degree equations," roughly in the sense of multiplications $V \otimes V \otimes \ldots \otimes V \rightarrow H^{0}(n \mathcal{L})$.

Remark 3.12. Like our previous algorithms, Theorem/Algorithm 3.10 runs in time $O\left(\delta^{4}\right)$, in the notation of Remark 3.8. This is because the "multiplication" involved in computing $\mu(f \otimes V)$ takes $O\left(\delta^{3}\right)$ steps, including reducing the basis 
$\left\{\mu\left(f \otimes t_{i}\right)\right\}$ for $H^{0}\left(2 \mathcal{L}-D-D^{\prime}\right)$ to echelon form. (We already know that the vectors $\left\{\mu\left(f \otimes t_{i}\right)\right\}$ are linearly independent.) It then takes another $O\left(\delta^{4}\right)$ steps to implement the second "division" step of Theorem/Algorithm [3.10, by the same discussion as in Remark 3.8 .

Now that we have discussed the flipping algorithm, we use a slight variant of it to give a second algorithm to compute the sum of two divisors. It is not clear whether this is more efficient than Theorem/Algorithm 3.6, as the new algorithm still requires time $O\left(\delta^{4}\right)$, although it involves slightly different linear algebra. On the other hand, it has slightly weaker hypotheses, and can work in the setting of Section 4 with $d_{0}=2 g$.

Theorem/Algorithm 3.13 (Addition of divisors (second method)). Let $D$ and $E$ be two divisors with $2 g \leq d \leq N-2 g$. (We do not make any assumptions on e.) Then we can compute $W_{D+E}=H^{0}(\mathcal{L}-D-E)$ as follows:

(1) Choose a nonzero $f \in W_{D}$, whose divisor (viewing $f$ as a section of $\mathcal{L}$ ) is $(f)=D+D^{\prime}$; proceed as in Theorem/Algorithm 3.10 to compute $W_{D^{\prime}}$.

(2) Also compute $H^{0}\left(2 \mathcal{L}-D-D^{\prime}-E\right)=\mu\left(f \otimes W_{E}\right)$.

(3) Compute $W_{D+E}=\left\{s \in V \mid \forall t \in W_{D^{\prime}}, \quad \mu(s \otimes t) \in H^{0}\left(2 \mathcal{L}-D-D^{\prime}-E\right)\right\}$.

Proof. This is a direct adaptation of the argument in Theorem/Algorithm 3.10 The hypothesis on $d$ ensures that $W_{D}$ and $W_{D^{\prime}}$ have no base points. Note that, as in Theorem/Algorithm 3.6 we cannot hope to recover the actual divisor $D+E$ unless $d+e \leq N-2 g$.

We conclude this section with an algorithm which tells whether a given codimension $d$ subspace $W \subset V$ actually is of the form $W_{D}$ for a divisor $D$. In other words, given a point on the Grassmannian $\mathbf{G}_{d}(V)$, we wish to identify whether or not it lies in the image of $\operatorname{Sym}^{d}(C)$. Since we have not explicitly written down equations describing this subvariety of the Grassmannian, we have to do something cleverer than checking whether certain polynomial functions vanish. The answer turns out to be relatively straightforward, using Theorem/Algorithm 3.10. Once again, the algorithm takes time $O\left(\delta^{4}\right)$.

Theorem/Algorithm 3.14 (Membership test). Let $W \subset V=H^{0}(\mathcal{L})$ have codimension $d$, where $2 g \leq d \leq N-2 g$. (In particular, $N \geq 4 g$.) Choose any nonzero $f \in W$, and compute $W^{\prime}$ in the same way as in Theorem/Algorithm [3.10; i.e.,

$$
W^{\prime}=\{s \in V \mid \forall t \in W, \quad \mu(s \otimes t) \in \mu(f \otimes V)\} .
$$

Then $W$ is of the form $W_{D}$ for some (uniquely determined) $D$ if and only if the codimension of $W^{\prime}$ in $V$ is $N-d$, or equivalently if $\operatorname{dim} W^{\prime}=d+1-g$.

Proof. If $W$ is of the form $W_{D}$, then the resulting $W^{\prime}$ is equal to the space $W_{D^{\prime}}$ in Theorem/Algorithm 3.10, where the degree of $D^{\prime}$ is $d^{\prime}=N-d$. Then $\mathcal{L}-D^{\prime}$ has degree $d$, and the result follows. Conversely, given any $W$, define a divisor $D=\sum m_{P} P$ by the property that for each $P, m_{P}$ is the minimum order to which any nonzero section $s \in W$ vanishes at $P$ (viewing $s$ as a section of $\mathcal{L}$ ). In other words, $D$ is the largest possible divisor for which $W \subset W_{D}$, and $D$ is the only possible candidate for a divisor which might give rise to $W$. (One easily checks that $D$ is defined over $k$, even if the individual points are only defined over $\bar{k}$, but we do not actually need this fact for our proof, and give our proof by working entirely over $\bar{k}$. As usual, there is no problem in extending scalars from $k$ to $\bar{k}$ for the proof.) 
Note that we do not yet know whether $\operatorname{deg} D=d$, in contrast to our previous notational convention. We do know, however, that $\operatorname{deg} D \leq d$, since otherwise, by Lemma [2.1, the codimension of $W_{D}$ would be at least $d+1$, contradicting the inclusion $W \subset W_{D}$. We see in fact that $\operatorname{deg} D=\operatorname{codim} W_{D} \leq d=\operatorname{codim} W$, and that $W=W_{D}$ if and only if $\operatorname{deg} D=d$.

Now our choice of $f$ gives us in particular a nonzero element of $W_{D}$, so the divisor of $f$ is of the form $D+D^{\prime}$, where

$$
d^{\prime}=\operatorname{deg} D^{\prime}=N-\operatorname{deg} D \geq N-d .
$$

The same reasoning as in Lemma/Algorithm 2.3 then shows us that $W^{\prime}=W_{D^{\prime}}$. The main point is that $\mu(f \otimes V)=H^{0}\left(2 \mathcal{L}-D-D^{\prime}\right)$, just as in Theorem/Algorithm 3.10 on the other hand, for each $P$, we can already find a section $t \in W$ that vanishes at $P$ precisely to the minimum possible order $m_{P}$. Now suppose that $\operatorname{deg} D^{\prime}$ were different from $N-d$. In that case, equation (3.11) would imply that $d^{\prime} \geq N-d+1$. Let $E$ be the sum of any $N-d+1$ points of $D^{\prime}$; then the codimension of $W_{D^{\prime}}$ in $V$ would be greater than or equal to the codimension of $W_{E}$, which we know to be $N-d+1$ (we are using the fact that $N-d+1 \leq N-2 g+1$, so as to apply Lemma 2.1). This would contradict our assumption on the codimension of $W_{D^{\prime}}$; hence we conclude that $\operatorname{deg} D^{\prime}=N-d$ after all, and hence by (3.11) we conclude that $\operatorname{deg} D=d$ and that $W=W_{D}$, as desired.

\section{The large MOdel: Weierstrass-Style AlgorithmS ON DIVISOR CLASSES AND ON THE JACOBIAN}

We now describe a way to work with divisor classes on $C$, which is inspired by the usual geometric definition of the group law on an elliptic curve represented by the plane cubic equation $y^{2}=x^{3}+a x+b$. Actually, in many cases, we give two algorithms for the basic operations, the first of which is straightforward and elegant, and the second of which is somewhat more efficient, and is given to illustrate various techniques. In Section 5 we give slightly faster algorithms, using a somewhat more compact representation of divisor classes. In all cases, the various algorithms require $O\left(\delta^{4}\right)$ operations in the field $k$, where $\delta=\operatorname{dim} V$ is approximately $5 g$. The main improvement in speed in Section 5 comes from our bringing $\delta$ down to a smaller multiple of $g$ there. Thus the algorithms in Section 5 supersede the ones here, at the expense of elegance. The basic structure of all the algorithms is roughly the same in all settings. We refer to the setup in this section as the large model.

We write $D \sim E$ to denote that the divisors $D$ and $E$ are linearly equivalent, and we denote the equivalence class of $D$ by $[D]$. We keep the notation of Section 3 , and specialize the situation to our application as follows. Take an effective divisor $D_{0}$ of degree $d_{0} \geq 2 g$ (or $2 g+1$, if we wish to repeatedly use Theorem/Algorithm 3.6 instead of 3.13). Fix the ambient line bundle $\mathcal{L}=\mathcal{O}_{C}\left(3 D_{0}\right)$; thus $N=3 d_{0} \geq 6 g$. Not surprisingly, we also assume that $g \geq 1$ (but everything works with $g=0$ and $d_{0} \geq 1$, if one is desperate or perverse enough to use these algorithms in that case). In the best case, $d_{0}=2 g$, and $\operatorname{dim} V=\delta=N+1-g=5 g+1$. In practice, it may be tricky to find $D_{0}$ of a specific degree, unless the curve $C$ contains one or more explicitly known rational points. If so, one may need to use a slightly larger value of $d_{0}$.

An element of the Jacobian of $C$ is a linear equivalence class of degree zero divisors; these can all be obtained as classes of the form $\left[D-D_{0}\right]$, where $D$ is 
an effective divisor of degree $d_{0}$, called a small divisor. Given such a divisor, it corresponds to the element $x_{D}$ of the Jacobian, given by

$$
x_{D}=\left[D-D_{0}\right], \quad \text { which is represented by the subspace } W_{D} \subset V .
$$

(Thus, if $d_{0}=2 g$, then $W_{D}$ is a $(3 g+1)$-dimensional subspace of $V$.) We shall also occasionally make use of large divisors, namely, divisors of degree $2 d_{0}$, for intermediate results in computations. We have already addressed in Theorem/Algorithm 3.14 the question of how to test whether a subspace $W \subset V$ actually corresponds to an element of the Jacobian. We now turn to the question of deciding when two such subspaces, of the form $W_{D}$ and $W_{E}$, represent the same element of the Jacobian. This amounts to testing whether the divisors $D$ and $E$ are linearly equivalent.

Theorem/Algorithm 4.1 (Equality of divisor classes). Let $D$ and $E$ be divisors of the same degree $d \leq N-2 g$; typically, they will be either both small, or both large divisors, represented by $W_{D}$ and $W_{E}$. Choose any nonzero section $f \in W_{D}$, whose divisor (viewing $f$ as a section of $\mathcal{L}$ ) is $(f)=D+D^{\prime} \sim \mathcal{L} \sim 3 D_{0}$. Then compute $W_{D^{\prime}+E}$ in one of the following two ways:

- Use Theorem/Algorithm 3.10 to compute the space $W_{D^{\prime}}$, and then compute $W_{D^{\prime}+E}$ by Theorem/Algorithm 3.6 or 3.13. This is less efficient but easier to explain than the method proposed below. However, this first method further requires the assumption that $2 g+1 \leq d \leq N-2 g-1$, in case we use Theorem/Algorithm 3.6. If we use Theorem/Algorithm 3.13 instead, then we can weaken the assumption to $2 g \leq d \leq N-2 g$.

- Alternatively, adapt the method of Theorem/Algorithm 3.13 without explicitly calculating $W_{D^{\prime}}$. Namely, calculate $H^{0}\left(2 \mathcal{L}-D-D^{\prime}-E\right)=\mu\left(f \otimes W_{E}\right)$. Then $W_{D^{\prime}+E}=\left\{s \in V \mid \forall t \in W_{D}, \quad \mu(s \otimes t) \in H^{0}\left(2 \mathcal{L}-D-D^{\prime}-E\right)\right\}$.

Then, in either case, $E \sim D$ (i.e., $[E]=[D]$, meaning $x_{E}=x_{D}$ on the Jacobian) if and only if the space $W_{D^{\prime}+E}$ is not zero, in which case it must be one-dimensional.

Proof. The second method to calculate $W_{D^{\prime}+E}$ works by the same reasoning as in the proof of Theorem/Algorithm 3.13. Now if there exists a nonzero $s \in W_{D^{\prime}+E}$, then its divisor of zeros $(s)$ (viewing $s$ as a section of $\mathcal{L}$ ) is an effective divisor of degree $N$ which contains $D^{\prime}+E$. By comparing degrees, we see that $D^{\prime}+E=(s)$. However, $(s)$ and $(f)$ are linearly equivalent (both belong to the class of $\mathcal{L}$ ), from which we obtain $E \sim D$. Conversely, if we have $E \sim D$, then $D^{\prime}+E \sim \mathcal{L}$, and it follows that $W_{D^{\prime}+E}=H^{0}\left(\mathcal{O}_{C}\left(\mathcal{L}-D^{\prime}-E\right)\right) \cong H^{0}\left(\mathcal{O}_{C}\right)=k$.

Remark 4.2. In the first method above, instead of using Theorem/Algorithm 3.6 or 3.13 to compute $W_{D^{\prime}+E}$, we can choose the section $f$ randomly, in which case there is a high probability that the divisors $D^{\prime}$ and $E$ are disjoint. In that case, we simply check if there are any nonzero elements in $W_{D^{\prime}} \cap W_{E}=W_{D^{\prime}+E}$. As mentioned in Remark 3.8 it may not be worth our while to test whether $D^{\prime}$ and $E$ are disjoint; however, if the intersection is zero, then we can at least immediately conclude that $x_{E} \neq x_{D}$, which saves a good amount of time. Another point to consider is that if we wish to test the equality of many different classes $[E]$ against $[D]$ by the first method, we should probably compute $W_{D^{\prime}}$ just once, instead of choosing a random $f$ each time. We note incidentally that the space $W_{D^{\prime}+E}$ that we compute has codimension less than the degree of $D^{\prime}+E$, a fact that follows immediately from the fact that $W_{D^{\prime}+E}$ is either zero or one-dimensional. We refer the reader to Remark [3.7] for some further discussion. 
We now describe the main building block in our algorithms to effectively compute in the Jacobian of $C$. This algorithm is analogous to the main geometric construction on the Weierstrass model of an elliptic curve in the plane. Namely, given two points $P_{1}, P_{2}$ on the elliptic curve, we draw the line through them and find its third point of intersection $Q$ with the curve. This means that $[Q]=-\left(\left[P_{1}\right]+\left[P_{2}\right]\right)$ in the group law on the elliptic curve. We generalize this to the case of divisors of degree $d_{0}$ on our curve $C$.

Proposition/Algorithm 4.3 ("Addflip"). Consider two subspaces $W_{D_{1}}, W_{D_{2}}$ associated to two small divisors $D_{1}, D_{2}$ on $C$, corresponding to the classes $x_{D_{1}}, x_{D_{2}}$ on the Jacobian. The following algorithm then computes the subspace $W_{E}$ associated to a divisor $E$ such that $x_{E}=-\left(x_{D_{1}}+x_{D_{2}}\right)$ (i.e., $\left.x_{D_{1}}+x_{D_{2}}+x_{E}=0\right)$ :

(1) First compute $W_{D_{1}+D_{2}}$, either by Theorem/Algorithm [3.6] or [3.13, or if possible by part 1 of Proposition/Algorithm 3.4.

(2) Now apply Theorem/Algorithm [3.10 to obtain the space $W_{E}$ corresponding to a "flip" $E$ of $D_{1}+D_{2}$, where $D_{1}+D_{2}+E \sim 3 D_{0}$.

Proof. Immediate. Note that step 1 is analogous to drawing the line through two points on a Weierstrass model elliptic curve in the plane, and step 2 is analogous to finding the third point on the intersection of the elliptic curve with the line. Also note that in step 1, our use of Theorem/Algorithm 3.6 or 3.13 means that we do not need to give special treatment to the case where $D_{1}=D_{2}$. In the Weierstrass model, this would mean that our line through a double point is automatically calculated as the appropriate tangent line. It is however simpler in the Weierstrass model to find the line through two distinct points. The analogous statement in our situation is: if we know beforehand that $D_{1}$ and $D_{2}$ are disjoint, then we can avoid using the more complicated theorems/algorithms, and instead simply calculate $W_{D_{1}+D_{2}}=W_{D_{1}} \cap W_{D_{2}}$ as in Proposition/Algorithm 3.4 This will save a lot of time. In practice, the divisors $D_{1}$ and $D_{2}$ are likely to be disjoint, so one should first calculate the intersection, and only use the more complicated algorithms for adding divisors in case the codimension of $W_{D_{1}} \cap W_{D_{2}}$ is not $2 d_{0}$.

Now that we have implemented the "addflip" operation $f\left(x_{1}, x_{2}\right)=-\left(x_{1}+x_{2}\right)$ on the Jacobian of $C$, we can describe algorithms for all the group operations. We begin with negation. Given a small divisor $D$, we seek $E$ such that $x_{E}=-x_{D}$, or in other words $D+E \sim 2 D_{0}$. This can be done in a straightforward way, but we give a slight modification which uses vector spaces of smaller dimension. This will make the negation algorithm more efficient, a point especially worth noting, since Theorem/Algorithm 4.5 (which adds two points on the Jacobian) involves a negation. Similar ideas also come into play for subtraction in the Jacobian, as discussed in Proposition/Algorithm 4.6

Theorem/Algorithm 4.4 (Negation). Let $W_{D}$ be given, where $D$ is a small divisor. Then we can compute $W_{E}$, where $x_{E}=-x_{D}$, in either one of the following two ways:

- Apply Proposition/Algorithm 4.3 to $D_{1}=D$ and $D_{2}=D_{0}$. We of course keep the space $W_{D_{0}}$ at hand; it corresponds to the zero element on the Jacobian.

- Alternatively, if one is willing to hold in memory more extensive information on the multiplication map $\mu$, one can find $W_{E}$ using vector spaces of 
smaller dimension. Namely, we need to know the multiplication maps $\mu_{21}$ and $\mu_{32}$, where we write $\mu_{m n}$ for the map

$$
\mu_{m n}: H^{0}\left(\mathcal{O}_{C}\left(m D_{0}\right)\right) \otimes H^{0}\left(\mathcal{O}_{C}\left(n D_{0}\right)\right) \rightarrow H^{0}\left(\mathcal{O}_{C}\left((m+n) D_{0}\right)\right) .
$$

We then proceed as follows:

(1) With respect to $\mu_{21}$, compute the space $H^{0}\left(\mathcal{O}_{C}\left(2 D_{0}-D\right)\right)$ as

$$
\left\{s \in H^{0}\left(\mathcal{O}_{C}\left(2 D_{0}\right)\right) \mid \forall t \in H^{0}\left(\mathcal{O}_{C}\left(D_{0}\right)\right), \quad \mu_{21}(s \otimes t) \in W_{D}\right\} .
$$

(2) Choose any nonzero $f \in H^{0}\left(\mathcal{O}_{C}\left(2 D_{0}-D\right)\right)$, and note that $(f)=$ $D+E \sim 2 D_{0}$ (viewing $f$ as a section of $\mathcal{O}_{C}\left(2 D_{0}\right)$ ). Then using $\mu_{32}$, compute

$$
H^{0}\left(\mathcal{O}_{C}\left(5 D_{0}-D-E\right)\right)=\mu_{32}\left(H^{0}\left(\mathcal{O}_{C}\left(3 D_{0}\right)\right) \otimes f\right)=\mu_{32}(V \otimes f) .
$$

(3) Now use $\mu_{32}$ again to compute $W_{E}=H^{0}\left(\mathcal{O}_{C}\left(3 D_{0}-E\right)\right)$ as

$$
\left\{s \in V \mid \forall t \in H^{0}\left(\mathcal{O}_{C}\left(2 D_{0}-D\right)\right), \quad \mu_{32}(s \otimes t) \in H^{0}\left(\mathcal{O}_{C}\left(5 D_{0}-D-E\right)\right)\right\} .
$$

Proof. The first method proposed is completely straightforward. As for justifying the second method, we note that the assertion in step 1 follows from the usual argument, since our requirement that $d_{0} \geq 2 g$ implies that $H^{0}\left(\mathcal{O}_{C}\left(D_{0}\right)\right)$ has no base points. Equation (4.4) is analogous to the identity in step 1 of Theorem/Algorithm 3.10. Finally, the assertion in step 3 follows since $H^{0}\left(\mathcal{O}_{C}\left(2 D_{0}-D\right)\right)$ also has no base points.

Our last major algorithm is to add two divisor classes on the Jacobian; by now, the description is almost trivial. We note a similar algorithm for subtraction, which unexpectedly is slightly more efficient than the addition algorithm.

Theorem/Algorithm 4.5 (Addition on the Jacobian). Given small divisors $D_{1}$ and $D_{2}$, or rather their corresponding subspaces $W_{D_{1}}$ and $W_{D_{2}}$, we can compute a space $W_{E}$ corresponding to a divisor $E$ with $x_{E}=x_{D_{1}}+x_{D_{2}}$ (i.e., $E \sim D_{1}+D_{2}-D_{0}$ ) as follows:

(1) First use Proposition/Algorithm 4.3 to compute the space $W_{E^{\prime}}$, where $x_{E^{\prime}}=$ $-\left(x_{D_{1}}+x_{D_{2}}\right)$.

(2) Now use Theorem/Algorithm 4.4 to find a negation $E$ of $E^{\prime}$.

Proposition/Algorithm 4.6 (Subtraction). Given $D_{1}$ and $D_{2}$, we can find $E$ such that $x_{D_{1}}-x_{D_{2}}=x_{E}$ in either one of the following two ways:

- First negate $D_{1}$, thereby obtaining a small divisor $D^{\prime}$ with $x_{D^{\prime}}=-x_{D_{1}}$. Then apply Proposition/Algorithm 4.3 to $D^{\prime}$ and $D_{2}$. This yields $W_{E}$, where $x_{E}=-\left(-x_{D_{1}}+x_{D_{2}}\right)$ as desired. Note that this method only involves one negation, instead of the two negations that would be involved if we first negated $D_{2}$, and then used the addition algorithm.

- Alternatively, we take the operations in Theorems/Algorithms 4.4 and 3.13, and remove certain redundant steps to streamline the subtraction algorithm. This amounts to the following steps:

(1) Compute $H^{0}\left(\mathcal{O}_{C}\left(2 D_{0}-D_{1}\right)\right)$ by "dividing out" $D_{0}$, as in step 1 of Theorem/Algorithm 4.4. 
(2) Take a nonzero $f \in H^{0}\left(\mathcal{O}_{C}\left(2 D_{0}-D_{1}\right)\right)$, and write $(f)=D_{1}+D_{1}^{\prime}$, where we view $f$ as a section of $H^{0}\left(\mathcal{O}_{C}\left(2 D_{0}\right)\right)$. Then use $\mu_{32}$ as in step 2 of Theorem/Algorithm 4.4 to calculate the space $W^{\prime}$ given by

$$
W^{\prime}=H^{0}\left(\mathcal{O}_{C}\left(5 D_{0}-D_{1}-D_{1}^{\prime}-D_{2}\right)\right)=\mu_{32}\left(W_{D_{2}} \otimes f\right) .
$$

(3) Analogously to step 3 in Theorem/Algorithm 4.4. compute the space $H^{0}\left(\mathcal{L}-D_{1}^{\prime}-D_{2}\right)=H^{0}\left(\mathcal{O}_{C}\left(3 D_{0}-D_{1}^{\prime}-D_{2}\right)\right)$ as $\left\{s \in V \mid \forall t \in H^{0}\left(\mathcal{O}_{C}\left(2 D_{0}-D_{1}\right)\right), \quad \mu_{32}(s \otimes t) \in W^{\prime}\right\}$.

(4) Finally, apply Theorem/Algorithm 3.10 to obtain $W_{E}$ as the "flip" of the space $H^{0}\left(\mathcal{L}-D_{1}^{\prime}-D_{2}\right)$.

Proof. The first method is completely straightforward. As for the second, it boils down to the facts that $x_{D_{1}^{\prime}}=-x_{D_{1}}$ and that $x_{E}=-\left(x_{D_{1}^{\prime}}+x_{D_{2}}\right)$.

\section{Further improvements to the Algorithms in Section 4 THE MEDIUM AND SMALL MODELS}

We now sketch two ways to modify the algorithms from the previous section, while speeding up the algorithms by a constant factor, so the asymptotics of our algorithms become a smaller multiple of $g^{4}$. In both cases, we achieve this linear speedup by reducing the degree of the basic line bundle $\mathcal{L}$, and with it the dimension of the ambient space $V$.

We shall refer to our first method as the medium model. We fix as before an effective divisor $D_{0}$ of degree $d_{0} \geq 2 g+1$. (It is possible, but a bit awkward, to work with $d_{0}=2 g$, but this would hamper us in our liberal use of Lemma 2.2.) We let $\mathcal{L}=\mathcal{O}_{C}\left(2 D_{0}\right)$, and represent a point $x_{D}=\left[D-D_{0}\right]$ by the space $W_{D}$, as before. Here $\operatorname{deg} D=d_{0}$; in case $d_{0}=2 g+1$, we obtain that $W_{D}$ is a $(g+2)$ dimensional subspace of the $(3 g+3)$-dimensional space $V=H^{0}\left(\mathcal{O}_{C}\left(2 D_{0}\right)\right)$. Since $\delta=\operatorname{dim} V=3 g+3$ in this case, we have improved the quantity $\delta^{4}$ by a factor of around $(5 / 3)^{4} \approx 7.7$ over the previous section; this is offset by the fact that we now have to do about twice as much linear algebra per operation in the Jacobian. So we predict an effective speedup by approximately a factor of 4 .

Our first observation regarding the medium model is that we can still use Theorems/Algorithms 3.14 and 4.1 to test for membership of and equality in the Jacobian in the medium model. We also observe that negation is now simply a matter of "flipping," as in Theorem/Algorithm 3.10. We need to make some modifications to the addition and subtraction algorithms, though. We first give the algorithm for the "addflip" operation, analogously to Proposition/Algorithm 4.3 The idea is virtually the same, but we need to keep track of more multiplication maps. We shall generally use the notation $\mu_{m n}$ to denote the same multiplication map as in equation (4.2):

$$
\mu_{m n}: H^{0}\left(m D_{0}\right) \otimes H^{0}\left(n D_{0}\right) \rightarrow H^{0}\left((m+n) D_{0}\right) .
$$

Note that we have simplified notation in this section by writing $m D_{0}$ instead of, say, $\mathcal{O}_{C}\left(m D_{0}\right)$.

Proposition/Algorithm 5.1 (Addflip (medium model)). Given the spaces $W_{D_{1}}=$ $H^{0}\left(2 D_{0}-D_{1}\right)$ and $W_{D_{2}}=H^{0}\left(2 D_{0}-D_{2}\right)$, we calculate the space $W_{E}$, corresponding to $x_{E}=-\left(x_{D_{1}}+x_{D_{2}}\right)$, as follows:

(1) Compute $H^{0}\left(4 D_{0}-D_{1}-D_{2}\right)=\mu_{22}\left(W_{D_{1}} \otimes W_{D_{2}}\right)$, by Lemma 2.2 
(2) Compute $H^{0}\left(3 D_{0}-D_{1}-D_{2}\right)=\left\{s \in H^{0}\left(3 D_{0}\right) \mid \forall t \in H^{0}\left(D_{0}\right), \quad \mu_{31}(s \otimes t) \in\right.$ $\left.H^{0}\left(4 D_{0}-D_{1}-D_{2}\right)\right\}$.

(3) Take a nonzero $f \in H^{0}\left(3 D_{0}-D_{1}-D_{2}\right)$; viewing $f$ as a section of $\mathcal{O}_{C}\left(3 D_{0}\right)$, we obtain $(f)=D_{1}+D_{2}+E$, where $x_{E}$ is the desired class. Now compute $H^{0}\left(5 D_{0}-D_{1}-D_{2}-E\right)=\mu_{32}\left(f \otimes H^{0}\left(2 D_{0}\right)\right)$.

(4) The desired result is $W_{E}=\left\{s \in V \mid \forall t \in H^{0}\left(3 D_{0}-D_{1}-D_{2}\right), \quad \mu_{23}(s \otimes t) \in\right.$ $\left.H^{0}\left(5 D_{0}-D_{1}-D_{2}-E\right)\right\}$.

The addition algorithm is then obtained by performing an addflip, followed by a regular flip to negate the result. Subtraction can be done similarly to the first method of Proposition/Algorithm 4.6. Alternatively, one can adapt the second method from Proposition/Algorithm 4.6, step 1 in that setting is superfluous, and one can obtain $H^{0}\left(3 D_{0}-D_{2}\right)=\mu_{21}\left(W_{D_{2}} \otimes H^{0}\left(D_{0}\right)\right)$ for use in step 2 there, and continue with step 3 . Then modify step 4 of that algorithm to resemble the last two steps of Proposition/Algorithm 5.1 We have described the alternative subtraction algorithm to illustrate possible techniques, but the simpler subtraction is probably just as quick.

We next turn to what we shall call the small model. In principle, one can represent all points on the Jacobian using divisors of degree $g$, which can be uniquely described using an ambient line bundle of degree $3 g$. However, in order to use Lemma 2.2 and to avoid some contortions, we shall use instead a divisor $D_{0}$ of degree $d_{0}=g+1$ (or at least $d_{0} \geq g+1$ ), and put $\mathcal{L}=\mathcal{O}_{C}\left(3 D_{0}\right)$. So in this case $V=H^{0}\left(\mathcal{O}_{C}\left(3 D_{0}\right)\right)$ has dimension $2 g+4$, but we need to go up to at least $H^{0}\left(7 D_{0}\right)$ within our calculation. We use the same notation for $\mu$ as in (5.1). The algorithms in this setting are reasonably direct generalizations of those in Section 4 and of those in the medium model; the main problem is that a divisor $D^{\prime}$ of degree $2 d_{0}$ may not be recognizable from the space $H^{0}\left(\mathcal{L}-D^{\prime}\right)$, which may have base points; so we need to work a bit more to deal with $D^{\prime}$ by looking instead at $H^{0}\left(\mathcal{L}^{\prime}-D^{\prime}\right)$ for a line bundle $\mathcal{L}^{\prime}$ of sufficiently high degree. On the other hand, the small model uses smaller-dimensional vector spaces and should give the fastest algorithms among the ones discussed in this paper. In the small model, we can test equality on the Jacobian using the second method of Theorem/Algorithm 4.1 (the reader is invited to find an analog of the first method, using techniques analogous to those in Propositions/Algorithms 5.2 [5.4]. We therefore content ourselves with the algorithms to test for membership of the Jacobian, for the addflip operation, and a slight improvement for negation (instead of reducing it to addflip). Addition and subtraction can then be done in a straightforward way, as in Section 4 . We note that one can obtain a slight improvement to the subtraction algorithm, similarly to the second method in Proposition/Algorithm 4.6. The details are slightly lengthy, and are left to the reader.

Proposition/Algorithm 5.2 (Membership test (small model)). Let $W \subset V$ be a codimension $d_{0}$ subspace. Then we can test whether $W=W_{D}$ for some $D$ as follows:

(1) Let $f$ be a nonzero element of $W$, and calculate $W_{+}=\mu_{43}\left(H^{0}\left(4 D_{0}\right) \otimes f\right) \subset$ $H^{0}\left(7 D_{0}\right)$.

(2) Calculate $W^{\prime}=\left\{s \in H^{0}\left(4 D_{0}\right) \mid \forall t \in W, \quad \mu_{43}(s \otimes t) \in W_{+}\right\}$.

(3) Then $W$ comes from a divisor $D$ if and only if $W^{\prime}$ has codimension $2 d_{0}$ in $H^{0}\left(4 D_{0}\right)$. 
Proof. This is entirely analogous to Theorem/Algorithm 3.14. The idea is that we hope to have $(f)=D+D^{\prime}$, whence $W_{+}=H^{0}\left(7 D_{0}-D-D^{\prime}\right)$ and $W^{\prime}=$ $H^{0}\left(4 D_{0}-D^{\prime}\right)$. We have had to use the higher-degree line bundle $\mathcal{O}_{C}\left(4 D_{0}\right)$ in addition to $\mathcal{L}$, in order to ensure that the degrees are large enough for us to be able to use Lemma/Algorithm 2.3 .

Proposition/Algorithm 5.3 (Addflip (small model)). Given the two subspaces $W_{D_{1}}=H^{0}\left(3 D_{0}-D_{1}\right)$ and $W_{D_{2}}=H^{0}\left(3 D_{0}-D_{2}\right)$, we can find $W_{E}$, where $x_{E}=$ $-\left(x_{D_{1}}+x_{D_{2}}\right)$, by modifying Proposition/Algorithm 4.3 as follows:

(1) Compute $H^{0}\left(3 D_{0}-D_{1}-D_{2}\right)$ by Theorem/Algorithm 3.6, without discarding the intermediate result $H^{0}\left(6 D_{0}-D_{1}-D_{2}\right)$. (It is a good idea to obtain $H^{0}\left(4 D_{0}-D_{1}-D_{2}\right)$ in the process; this can be done by setting up linear equations to simultaneously "divide" out both $H^{0}\left(3 D_{0}\right)$ and $H^{0}\left(2 D_{0}\right)$ from $H^{0}\left(6 D_{0}-D_{1}-D_{2}\right)$, the former via (3.4), and the latter via an analogous calculation that largely overlaps with (3.4).)

(2) Take a nonzero $f \in H^{0}\left(3 D_{0}-D_{1}-D_{2}\right)$; so, viewing $f$ as a section of $\mathcal{O}_{C}\left(3 D_{0}\right)$, we have $(f)=D_{1}+D_{2}+E$ for the desired $E$.

(3) Calculate $W_{E}$ as

(5.2) $\left\{s \in H^{0}\left(3 D_{0}\right) \mid \forall t \in H^{0}\left(6 D_{0}-D_{1}-D_{2}\right), \quad \mu_{36}(s \otimes t) \in \mu_{36}\left(f \otimes H^{0}\left(6 D_{0}\right)\right)\right\}$.

Note that this method involves calculation in $H^{0}\left(9 D_{0}\right)=H^{0}(3 \mathcal{L})$; if we have done the extra computation in step 1 , we can limit ourselves to $H^{0}\left(7 D_{0}\right)$, by replacing $6 D_{0}$ by $4 D_{0}$ throughout (5.2).

Proof. Immediate. Note the trouble that we went to because $H^{0}\left(3 D_{0}-D_{1}-D_{2}\right)$ is not necessarily base point free, which meant that we had to use (5.2) instead of doing a regular "flipping" algorithm after step 1 above. However, we still point out that, generically, $H^{0}\left(3 D_{0}-D_{1}-D_{2}\right)$ is base point free, since its degree is $d_{0} \geq g+1$.

Proposition/Algorithm 5.4 (Negation (small model)). Given $W_{D}$, we can find $W_{E}$, where $x_{E}=-x_{D}$, by using the addflip operation. Alternatively, we can use the following slightly faster method:

(1) Calculate $H^{0}\left(5 D_{0}-D\right)=\mu_{23}\left(H^{0}\left(2 D_{0}\right) \otimes W_{D}\right)$. Continue by calculating $H^{0}\left(2 D_{0}-D\right)=\left\{s \in H^{0}\left(2 D_{0}\right) \mid \forall t \in V, \quad \mu_{23}(s \otimes t) \in H^{0}\left(5 D_{0}-D\right)\right\}$.

(2) Take a nonzero $f \in H^{0}\left(2 D_{0}-D\right)$, where $(f)=D+E$. Then compute $H^{0}\left(6 D_{0}-D-E\right)=\mu_{24}\left(f \otimes H^{0}\left(4 D_{0}\right)\right)$.

(3) Compute $W_{E}=\left\{s \in H^{0}\left(3 D_{0}\right) \mid \forall t \in W_{D}, \quad \mu_{33}(s \otimes t) \in H^{0}\left(6 D_{0}-D-E\right)\right\}$.

Proof. This is just like Theorem/Algorithm4.4 but we have included the algorithm to show in step 1 the technique of going from $H^{0}\left(3 D_{0}-D\right)$ to $H^{0}\left(2 D_{0}-D\right)$ by virtue of first "going up," then "going down." This is because the degree of $D_{0}$ is too small for us to automatically be able to "divide" by $H^{0}\left(D_{0}\right)$. If we were originally careful enough to select $D_{0}$ so that $H^{0}\left(D_{0}\right)$ were base point free, we could have begun as in Theorem/Algorithm 4.4 However, we cannot in any way guarantee that $H^{0}\left(2 D_{0}-D\right)$ is base point free, since $D$ is arbitrary; hence, concluding with the computation in (4.5) was never an option in the setting of the small model.

We conclude this paper with a brief discussion of converting points on the Jacobian between their representations in the different models. The first observation is that it is easy to pass between two representations of the same (as always, effective) 
divisor $D$ in terms of spaces $W_{D}$ of sections of two different line bundles $\mathcal{L}_{1}$ and $\mathcal{L}_{2}$. (Typically, $\mathcal{L}_{1}=\mathcal{O}_{C}\left(n_{1} D_{0}\right)$ and $\mathcal{L}_{2}=\mathcal{O}_{C}\left(n_{2} D_{0}\right)$ for a fixed $D_{0}$ and integers $n_{1}, n_{2}$.) So the problem is to pass from a knowledge of $H^{0}\left(\mathcal{L}_{1}-D\right)$ to one of $H^{0}\left(\mathcal{L}_{2}-D\right)$. Let us assume for a moment that $\operatorname{deg} \mathcal{L}_{1} \geq 2 g+1+\operatorname{deg} D$. Then take auxiliary line bundles $\mathcal{L}_{1}^{\prime}, \mathcal{L}_{2}^{\prime}$ of degree at least $2 g+1$, such that $\mathcal{L}_{1}+\mathcal{L}_{1}^{\prime}=\mathcal{L}_{2}+\mathcal{L}_{2}^{\prime}$; here, addition of line bundles really refers to their tensor product. Then, using multiplication of sections in $H^{0}\left(\mathcal{L}_{1}-D\right)$ and $H^{0}\left(\mathcal{L}_{1}^{\prime}\right)$, we can obtain the space $H^{0}\left(\mathcal{L}_{1}+\mathcal{L}_{1}^{\prime}-D\right)=H^{0}\left(\mathcal{L}_{2}+\mathcal{L}_{2}^{\prime}-D\right)$. We can then "divide" by $H^{0}\left(\mathcal{L}_{2}^{\prime}\right)$ to obtain $H^{0}\left(\mathcal{L}_{2}-D\right)$, as desired. This is similar to step 1 in Proposition/Algorithm 5.4. Of course, if the degree of $\mathcal{L}_{1}$ is sufficiently larger than the degree of $\mathcal{L}_{2}$, we only need to perform a single division, without first raising the degree using $\mathcal{L}_{1}^{\prime}$. Similarly, if the degree of $\mathcal{L}_{2}$ is large compared to that of $\mathcal{L}_{1}$, then a single multiplication step is sufficient.

The above discussion seems to require the use of Lemma2.2 for the multiplication step. We can modify the procedure, though, to work whenever $H^{0}\left(\mathcal{L}_{1}-D\right)$ is base point free (such as when $\operatorname{deg} \mathcal{L}_{1} \geq 2 g+\operatorname{deg} D$ ). The idea is to take a nonzero section $f \in H^{0}\left(\mathcal{L}_{1}-D\right)$, whose divisor (viewing $f$ as a section of $\mathcal{L}_{1}$ ) is $D+D^{\prime}$. Here we caution that the degree of $D^{\prime}$ may be large, so that $H^{0}\left(\mathcal{L}_{1}-D^{\prime}\right)$ may well have base points. To get around this, we take an auxiliary line bundle $\mathcal{L}^{\prime}$ of degree at least $2 g+1+\operatorname{deg} D^{\prime}$, and multiply $f$ by $H^{0}\left(\mathcal{L}^{\prime}\right)$ to obtain $H^{0}\left(\mathcal{L}_{1}+\mathcal{L}^{\prime}-D-D^{\prime}\right)$. Dividing out $H^{0}\left(\mathcal{L}_{1}-D\right)$ then yields $H^{0}\left(\mathcal{L}^{\prime}-D^{\prime}\right)$. We can then multiply $f$ by $H^{0}\left(\mathcal{L}_{1}^{\prime}+\mathcal{L}^{\prime}\right)$, where $\mathcal{L}_{1}^{\prime}$ has suitably large degree, and then divide by $H^{0}\left(\mathcal{L}^{\prime}-D^{\prime}\right)$ (which is now base point free), to obtain $H^{0}\left(\mathcal{L}_{1}^{\prime}+\mathcal{L}_{1}-D\right)$ even in the case where we could not use Lemma 2.2 as in the above paragraph. We note that throughout all these computations, the degrees of the intermediate line bundles are at most $O\left(\max \left(\operatorname{deg} \mathcal{L}_{1}, \operatorname{deg} \mathcal{L}_{2}, g\right)\right)$.

Now that we have full control over the ambient line bundle $\mathcal{L}$ used to represent individual divisors $D$, we shall always assume that the degree of $\mathcal{L}$ is sufficiently large, and use $\mathcal{L}$ to represent all divisors (so $D$ is given by $W_{D}=H^{0}(\mathcal{L}-D$ ), unless stated otherwise). We now discuss how to change the basepoint $D_{0}$ used to map a divisor $D$ to the point $x_{D}=\left[D-D_{0}\right]$ on the Jacobian. It is always easy to add an effective divisor $E$ to $D_{0}$, since $\left[D-D_{0}\right]=\left[(D+E)-\left(D_{0}+E\right)\right]$, so we can use Theorem/Algorithm 3.6 to replace $W_{D}$ with $W_{D+E}$. In particular, we can also assume that $\operatorname{deg} D_{0} \geq 2 g+1$. Thus the main question that remains is how to replace $D_{0}$ by a new basepoint $E_{0}$ of smaller degree. This means that given $D$, we wish to find $E$ such that $\left[D-D_{0}\right]=\left[E-E_{0}\right]$; in other words, we seek an effective divisor $E \sim D+E_{0}-D_{0}$. Writing $D_{1}=D+E_{0}$ (which we compute as usual by Theorem/Algorithm 3.6, and whose degree is even larger than $\operatorname{deg} D=\operatorname{deg} D_{0} \geq 2 g+1$ ), we have reduced our question to the following result, which leads to an efficient algorithm for the Riemann-Roch problem on $C$. Like our other algorithms, this one requires $O\left(\max \left(\operatorname{deg} D_{0}, \operatorname{deg} D_{1}, g\right)^{4}\right)$ field operations in $k$. Remember that there is no loss of generality in letting $\mathcal{L}$ have large degree $N \geq 2 g+1+\operatorname{deg} D_{1}$.

Theorem/Algorithm 5.5 (Riemann-Roch). Let $\operatorname{deg} \mathcal{L}=N$, and assume given (effective) divisors $D_{1}$ and $D_{0}$, whose degrees lie between $2 g+1$ and $N-2 g-1$. Then we can compute the space $H^{0}\left(D_{1}-D_{0}\right)$, and with it (if the space is nonzero) an effective divisor $E \sim D_{1}-D_{0}$. The procedure is: 
(1) Use Theorem/Algorithm [3.10 to compute $D_{1}^{\prime}$ (specifically, compute $W_{D_{1}^{\prime}}=$ $H^{0}\left(\mathcal{L}-D_{1}^{\prime}\right)$ ), where $D_{1}+D_{1}^{\prime} \sim \mathcal{L}$. Thus $\mathcal{L}-D_{1}^{\prime}-D_{0} \sim D_{1}-D_{0}$, and an explicit isomorphism from $H^{0}\left(D_{1}-D_{0}\right)$ to $H^{0}\left(\mathcal{L}-D_{1}^{\prime}-D_{0}\right)$ is given by multiplication by the section $f$ of $\mathcal{L}$ with $(f)=D_{1}+D_{1}^{\prime}$ used in the calculation of $W_{D_{1}^{\prime}}$.

(2) Compute $H^{0}\left(2 \mathcal{L}-D_{1}^{\prime}-D_{0}\right)=\mu\left(W_{D_{1}^{\prime}} \otimes W_{D_{0}}\right)$, and use it to compute $W_{D_{1}^{\prime}+D_{0}} \cong H^{0}\left(D_{1}-D_{0}\right)$, as in Theorem/Algorithm 3.6.

(3) A divisor $E$ as above exists if and only if we can find a nonzero $g \in W_{D_{1}^{\prime}+D_{0}}$, in which case we view $g$ as a section of $\mathcal{L}$ and see that its divisor is $(g)=$ $D_{1}^{\prime}+D_{0}+E$. Then calculate

(5.3) $W_{E}=\left\{s \in H^{0}(\mathcal{L}) \mid \forall t \in H^{0}\left(2 \mathcal{L}-D_{1}^{\prime}-D_{0}\right), \quad \mu^{\prime}(s \otimes t) \in \mu^{\prime}\left(g \otimes H^{0}(2 \mathcal{L})\right)\right\}$.

Here $\mu^{\prime}: H^{0}(\mathcal{L}) \otimes H^{0}(2 \mathcal{L}) \rightarrow H^{0}(3 \mathcal{L})$ is the multiplication map. (We did not use the notational convention of (5.1), since we are not assuming that $\mathcal{L}$ is a multiple of $D_{0}$.)

Proof. We know that $\mu^{\prime}\left(g \otimes H^{0}(2 \mathcal{L})\right)=H^{0}\left(3 \mathcal{L}-D_{1}^{\prime}-D_{0}-E\right)$, and our generous assumptions about degrees ensure that $H^{0}\left(2 \mathcal{L}-D_{1}^{\prime}-D_{0}\right)$ is base point free. Note that we do not assume that the smaller space $H^{0}\left(\mathcal{L}-D_{1}^{\prime}-D_{0}\right)$ is base point free; indeed, its degree is $\operatorname{deg} D_{1}-\operatorname{deg} D_{0}$, which may be quite small. The rest of the proof is standard by now.

\section{ACKNOWLEDGMENTS}

I am grateful to K. Murty for posing for me the question of whether one could work algorithmically with abelian varieties without using explicit equations. I would also like to thank M. Thaddeus for very useful conversations on linear systems, in particular, for his referring me to the article [Laz89], and for his suggestion to think primarily in terms of the space $W_{D}$ and sections of $\mathcal{L}$, rather than its dual $W_{D}^{*}$ and the projective embedding. My thanks go to C. Gasbarri for helpful comments and references. I also gratefully acknowledge the support of the Clay Mathematics Institute, which partially supported this research by funding me as a Clay Scholar in the summer of 2000, and which supported my participation in the Clay Mathematics Institute Introductory Workshop in Algorithmic Number Theory at MSRI in August 2000. Finally, I would like to thank the mathematics department at Columbia University for its hospitality at the time of the final revisions to this manuscript.

\section{REFERENCES}

[ACGH85] E. Arbarello, M. Cornalba, P. A. Griffiths, and J. Harris, Geometry of algebraic curves. Vol. I, Grundlehren der Mathematischen Wissenschaften [Fundamental Principles of Mathematical Sciences], vol. 267, Springer-Verlag, New York, 1985. MR 86h:14019

[ADH94] Leonard M. Adleman, Jonathan DeMarrais, and Ming-Deh Huang, A subexponential algorithm for discrete logarithms over the rational subgroup of the Jacobians of large genus hyperelliptic curves over finite fields, Algorithmic number theory "ANTS-I" (Ithaca, NY, 1994) (Leonard M. Adleman and Ming-Deh Huang, eds.), Lecture Notes in Comput. Sci., vol. 877, Springer, Berlin, 1994, pp. 28-40. MR 96b:11078

[BLR90] Siegfried Bosch, Werner Lütkebohmert, and Michel Raynaud, Néron models, Ergebnisse der Mathematik und ihrer Grenzgebiete (3) [Results in Mathematics and Related Areas (3)], vol. 21, Springer-Verlag, Berlin, 1990. MR 91i:14034

[Can87] David G. Cantor, Computing in the Jacobian of a hyperelliptic curve, Math. Comp. 48 (1987), no. 177, 95-101. MR 88f:11118 
[GH94] Phillip Griffiths and Joseph Harris, Principles of algebraic geometry, Wiley Classics Library (reprint of 1978 edition), John Wiley \& Sons Inc., New York, 1994. MR 95d:14001

[GPS02] S. D. Galbraith, S. M. Paulus, and N. P. Smart, Arithmetic on superelliptic curves, Math. Comp. 71 (2002), no. 237, 393-405 (electronic). MR 2002h:14102

[Har77] Robin Hartshorne, Algebraic geometry, Springer-Verlag, New York, 1977, Graduate Texts in Mathematics, No. 52. MR 57:3116

[Hes99] Florian Hess, Zur Divisorenklassengruppenberechnung in globalen Funktionenkörpern, Ph.D. thesis, Technische Universität Berlin, 1999, may be downloaded from the web at http://www.math.tu-berlin.de/ ${ }^{\sim}$ kant/publications/diss/diss_FH.ps.gz.

[Hes02] Florian Hess, Computing Riemann-Roch spaces in algebraic function fields and related topics, J. Symbolic Comput. 33 (2002), no. 4, 425-445.

[HI94] Ming-Deh Huang and Doug Ierardi, Efficient algorithms for the Riemann-Roch problem and for addition in the Jacobian of a curve, J. Symbolic Comput. 18 (1994), no. 6, 519-539. MR 96h:14077

[HS00] Ryuichi Harasawa and Joe Suzuki, Fast Jacobian group arithmetic on $C_{a b}$ curves, Algorithmic number theory "ANTS-IV" (Leiden, 2000) (Wieb Bosma, ed.), Lecture Notes in Comput. Sci., vol. 1838, Springer, Berlin, 2000, pp. 359-376. MR 2002f:11073

[Laz89] Robert Lazarsfeld, A sampling of vector bundle techniques in the study of linear series, Lectures on Riemann surfaces (Trieste, 1987) (M. Cornalba, X. Gomez-Mont, and A. Verjovsky, eds.), World Sci. Publishing, Teaneck, NJ, 1989, pp. 500-559. MR 92f: 14006

[Mil86] J. S. Milne, Jacobian varieties, Arithmetic geometry (Storrs, Conn., 1984) (Gary Cornell and Joseph H. Silverman, eds.), Springer, New York, 1986, pp. 167-212. MR 89b:14029

[Vol94] Emil J. Volcheck, Computing in the Jacobian of a plane algebraic curve, Algorithmic number theory "ANTS-I" (Ithaca, NY, 1994) (Leonard M. Adleman and Ming-Deh Huang, eds.), Lecture Notes in Comput. Sci., vol. 877, Springer, Berlin, 1994, pp. 221233. MR 96a:14033

Mathematics Department and Center for Advanced Mathematical Sciences, AmeriCan University of Beirut, Bliss Street, Beirut, Lebanon

E-mail address: kmakdisi@aub.edu.lb 